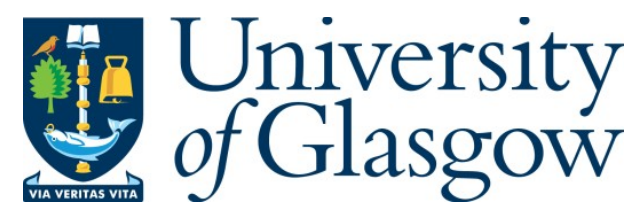

Agarwal, A. and Lorig, M. (2020) The implied Sharpe ratio. Quantitative Finance, 20(6), pp. 1009-1026.

(doi: 10.1080/14697688.2020.1718194)

This is the Author Accepted Manuscript.

There may be differences between this version and the published version. You are advised to consult the publisher's version if you wish to cite from it.

https://eprints.gla.ac.uk/207819/

Deposited on: 16 January 2019

Enlighten - Research publications by members of the University of Glasgow http://eprints.gla.ac.uk 


\title{
The implied Sharpe ratio
}

\author{
Ankush Agarwal * \\ Matthew Lorig ${ }^{\dagger}$ \\ January 15, 2020
}

\begin{abstract}
In an incomplete market, including liquidly-traded European options in an investment portfolio could potentially improve the expected terminal utility for a risk-averse investor. However, unlike the Sharpe ratio, which provides a concise measure of the relative investment attractiveness of different underlying risky assets, there is no such measure available to help investors choose among the different European options. We introduce a new concept - the implied Sharpe ratio - which allows investors to make such a comparison in an incomplete financial market. Specifically, when comparing various European options, it is the option with the highest implied Sharpe ratio that, if included in an investor's portfolio, will improve his expected utility the most. Through the method of Taylor series expansion of the state-dependent coefficients in a nonlinear partial differential equation, we also establish the behaviour of the implied Sharpe ratio with respect to an investor's risk-aversion parameter. In a series of numerical studies, we compare the investment attractiveness of different European options by studying their implied Sharpe ratio.
\end{abstract}

Keywords: Sharpe ratio, PDE asymptotics, stochastic volatility, Heston, reciprocal Heston

JEL classification code: G11

*Adam Smith Business School, University of Glasgow, G12 8QQ Glasgow, United Kingdom. e-mail: ankush.agarwal@glasgow.ac. uk

†Department of Applied Mathematics, University of Washington, Seattle, WA 98195, USA. e-mail: mlorig@uw.edu 


\section{Introduction}

The Sharpe ratio, defined as the ratio of a portfolio's expected return to its standard deviation, was introduced by Sharpe (1966) as a concise measure of mutual fund performance. Several other works have since used the idea under different settings to measure the performance of investment portfolios composed of risky assets. Jensen (1969) considered the effect of differential risk on required returns of different risky assets. Using a capital asset pricing model, he showed that the investment portfolios can be compared by looking at the difference between the actual returns on a portfolio and the expected returns on that portfolio conditional on its level of systematic risk and actual returns of the market portfolio. Another approach towards portfolio selection was proposed by Merton (1969) in which he incorporated the risk-preference of the investor through the risk-aversion parameter of a utility function. He considered an investor that trades risky assets in the market to maximise his expected terminal utility. Under the assumption that the log-asset returns are normally distributed with a constant Sharpe ratio, he showed that, assuming a constant relative risk-aversion utility function, the investor derives a higher expected utility from assets with higher Sharpe ratio.

Hodges and Neuberger (1989) used the idea of expected utility maximisation to create optimal replication portfolios for contingent claims in a market with transaction costs. Their work led to the idea of indifference pricing of contingent claims in incomplete markets (see Carmona (2008) for a collection of the related works). The framework of indifference pricing allows to identify an economically justifiable price of a contingent claim in an incomplete market. In such markets, it is not possible to perfectly replicate any contingent claim using a self-financing portfolio of the underlying risky assets and determine a unique price (see, for example, Section 10.3 Delbaen and Schachermayer (2006) for a complete mathematical characterisation of incomplete markets). Typically, the Hamilton-Jacobi-Bellman (HJB) equation satisfied by the indifference price is solved numerically. Recently, in an incomplete market framework based on a class of stochastic volatility models, Lorig (2018) approximately solved the HJB equation for the indifference price of a European option. He developed approximation techniques to solve nonlinear partial differential equations with state-dependent coefficients using a Taylor series expansion method developed in a series of papers: Pagliarani and Pascucci (2012), Lorig et al. (2015), Lorig et al. (2017). The corresponding approximate implied volatilities of the indifference prices are also provided by Lorig (2018).

As the underlying risky assets cannot be used to perfectly replicate a European option in an incomplete market, an investor looking to create an investment portfolio can benefit by including a European option in it. This raises an important question of how to measure the investment attractiveness of different European options available in the market. Similar to how the Sharpe ratio can be used to measure the performance of an underlying risky asset, in this paper we introduce the concept of an implied Sharpe ratio which can be viewed as a measure of a European option's worth to an investor. Typically, the value of a European option is quantified in terms of its implied volatility. When comparing two options, it is the option with a higher implied volatility that is considered to be the more expensive option. However, an option's implied volatility does not capture its worth to an investor. Relative to not owning the option, buying or selling an option at a given implied volatility may either increase or decrease an investor's expected terminal utility. The implied Sharpe ratio, as defined in this work, aims to address the issue of measuring an option's worth to an investor.

In the investment literature, the behavioural preference of an investor is captured through the risk-aversion parameter of his utility function. Many classical studies of investment under uncertainty derive comparative statics with respect to the risk-aversion parameter. For example, it is well established that a risk-averse investor is willing to pay a higher premium to insure himself against risk. Jewitt (1987) extended this classical comparative static of risk-aversion parameter in the presence of an extra source of uncertainty. Eeckhoudt et al. (1995) studied the impact of cost and price changes on the inventory of a risk-averse newsboy who must decide to purchase newspapers in order to sell them later. Similarly in our work, we aim to express the implied Sharpe ratio in terms of the model parameters and an investor's risk-aversion parameter in order to help him choose between European options with different strikes and times-to-maturity.

The rest of the paper is organised as follows: In Section 2, we define the implied Sharpe ratio and prove its uniqueness and existence in a general market setting. In Section 3, we introduce a general local stochastic volatility 
model which is popular among the practitioners and derive the equation for the implied Sharpe ratio in terms of the model parameters and risk-aversion parameter. In Section 4, we use a Taylor series expansion technique to develop semi-explicit approximations of the implied Sharpe ratio. In Section 5 we study the particular forms of implied Sharpe ratio using different examples of local stochastic volatility models and illustrate its importance in identifying the most suitable European option for investment among the available choices. Mathematical properties, proofs and a collection of formulas for calculating the implied Sharpe ratio under different models are presented in Appendices A-C.

\section{The implied Sharpe ratio}

Let us consider a frictionless financial market consisting of one risk-less asset with constant price and one nondividend paying risky asset represented by a real-valued semi-martingale $\mathrm{S}$ defined on a filtered probability space $\left(\Omega, \mathcal{F},\left(\mathcal{F}_{t}\right), \mathbb{P}\right)$ satisfying the usual conditions. Let $\mathrm{W}=\left(\mathrm{W}_{t}\right)_{0 \leq t \leq \mathrm{T}}$ denote the wealth process of an investor who invests in $\pi_{t}$ number of shares of $\mathrm{S}$ at time $t$ starting from initial wealth level $w \in \mathbb{R}$. Then, the wealth process $\mathrm{W}$ satisfies

$$
\mathrm{W}_{t}=w+\int_{0}^{t} \pi_{u} \mathrm{dS}_{u}
$$

where $\pi=\left(\pi_{t}\right)_{0 \leq t \leq \mathrm{T}}$ is a real-valued predictable process such that the stochastic integral above is well defined. In addition, we assume that the investor also owns European-style contingent claims on the risky asset. Over a fixed time horizon $\mathrm{T}>0$, the investor trades the risky asset in order to maximise his expected terminal utility. We suppose the investor's utility function $U: \mathbb{R} \rightarrow \mathbb{R}$ is strictly concave, strictly increasing and belongs to the class $C^{\infty}(\mathbb{R})$ of infinitely differentiable functions. The value of the investment to investor is captured by a function defined as follows:

Definition 1. The value function $\mathrm{V}$ of an investor with wealth process (1) and who owns $\nu$ European-style contingent claims, each with payoff function $\varphi\left(\mathrm{S}_{\mathrm{T}}\right)$, is defined as

$$
\mathrm{V}(t, s, w, \nu):=\sup _{\pi \in \Pi} \mathbb{E}_{t, s, w}\left[\mathrm{U}\left(\mathrm{W}_{\mathrm{T}}+\nu \varphi\left(\mathrm{S}_{\mathrm{T}}\right)\right)\right], \quad 0 \leq t<\mathrm{T}, s>0, w, \nu \in \mathbb{R}
$$

where $\Pi$ is the set of admissible strategies given as

$$
\Pi:=\left\{\pi:\left(\pi_{t}\right)_{0 \leq t \leq \mathrm{T}} \text { is predictable and } \mathbb{E}_{0, s, w} \int_{0}^{\mathrm{T}} \pi_{t}^{2} \mathrm{~d}\langle\mathrm{~S}, \mathrm{~S}\rangle_{t}<\infty\right\},
$$

and $\mathbb{E}_{t, s, w}[\cdot] \equiv \mathbb{E}\left[\cdot \mid \mathrm{S}_{t}=s, \mathrm{~W}_{t}=w\right]$ denotes conditional expectation under $\mathbb{P}$.

When $\nu<0$ the investor is a seller of the contingent claim, and when $\nu>0$ he is a buyer. If for a given European option payoff we can explicitly solve for V, we can find out whether the investor will prefer to sell or buy that European option. In an analogy to the Black-Scholes price of a European option, we define the Merton value function as the value function of an investor when we assume that the risky asset price follows a geometric Brownian motion.

Definition 2. Suppose the risky asset dynamics is given by the Black-Scholes model in which $\mathrm{dS}_{t}=\mu \mathrm{S}_{t} \mathrm{~d} t+$ $\sigma \mathrm{S}_{t} \mathrm{~dB}_{t}$, and where, $\mu \in \mathbb{R}$ is the expected rate of return, $\sigma \in \mathbb{R}_{+}$is the volatility and $\mathrm{B}=\left(\mathrm{B}_{t}\right)_{t \geq 0}$ is a standard Brownian motion. The Merton value function $\mathrm{V}^{\mathrm{M}}$ of an investor with wealth process (1) is then defined as

$$
\mathrm{V}^{\mathrm{M}}(t, w ; \lambda):=\sup _{\pi \in \Pi} \mathbb{E}_{t, s, w}\left[\mathrm{U}\left(\mathrm{W}_{\mathrm{T}}\right)\right]
$$

where $\lambda:=\frac{\mu}{\sigma}$ is the Sharpe ratio. 
The Merton value function is an increasing function with respect to $\lambda$. Thus, a risky asset with higher Sharpe ratio will lead to a higher Merton value function. Just as the implied volatility is used to link the market-observed European option price to the Black-Scholes model's European option price, we define the implied Sharpe ratio to link an investor's value function in a general risky-asset price model to the Merton value function in the BlackScholes model.

Definition 3. Suppose an investor with wealth process (1) owns $\nu$ European-style contingent claims with unit price $p$. Further suppose that $\mathrm{V}(t, s, w-\nu p, \nu) \geq \mathrm{U}(w)$ for all $t \in[0, \mathrm{~T}]$ and any $s>0, w, \nu \in \mathbb{R}$. Then, the implied Sharpe ratio is the unique positive solution $\Lambda$ of the equation

$$
\mathrm{V}^{\mathrm{M}}(t, w ; \Lambda)=\mathrm{V}(t, s, w-\nu p, \nu),
$$

where $\mathrm{V}^{\mathrm{M}}$ is the Merton value function and $\mathrm{V}$ is the investor's value function.

According to the above definition, if an investor assumes that the risky asset price follows a geometric Brownian motion with Sharpe ratio $\Lambda$ and invests only in that risky asset, he will obtain the same expected utility as he would by investing in a portfolio composed of the risky asset and a European option, in a general risky-asset price model. European calls and puts are usually compared based on their implied volatilities. When plugged in the Black-Scholes model's European call/put formula, implied volatility provides the market price of the option. However, it does not provide any measure of the option's worth to an investor who is looking to maximize his expected terminal utility. The implied Sharpe ratio rectifies this shortcoming of implied volatility.

Through the implied Sharpe ratio, we can connect an investor's value function V in an incomplete market to the classical Merton value function $\mathrm{V}^{\mathrm{M}}$. In doing so, our aim is two-fold: First, by computing the implied Sharpe ratio for $\nu>0$ (buying the option) or $\nu<0$ (selling the option), we can compare it to the case $\nu=0$ (no position in the option). From Definition 3, it is clear that a higher implied Sharpe ratio, compared to the other possibilities, will deliver a higher value to an investor who is looking to maximise his expected terminal utility over a finite time horizon. Thus, the implied Sharpe ratio can tell us whether buying $(\nu>0)$ or selling $(\nu<0)$ a contingent claim can improve the investor's utility as opposed to just investing in the risky asset $(\nu=0)$ underlying the option. This comparison can also be made directly by comparing the value functions corresponding to the different possibilities $(\nu<0, \nu>0, \nu=0)$. However, as we will see later, in the case of an exponential utility function, the implied Sharpe ratio is independent of the starting wealth level. Thus, it provides a standard measure of investment worth as opposed to the value function which is dependent on the starting level of wealth.

Second, by computing the implied Sharpe ratio for two European options with different strikes and maturities, we can compare their relative worth to an investor. The European option with the higher implied Sharpe ratio will be more attractive to the investor. Such a comparison can be made across the whole range of available European options, thus making the implied Sharpe ratio a better measure than the implied volatility to assess the worth of an option for investment. The implied volatility is a unitless quantity which provides a better comparison between different European options than their respective prices. In the same spirit, the implied Sharpe ratio allows a more intuitive comparison between the different European options than their respective expected utility values which is dependent on initial wealth. It is important to note that since we develop the implied Sharpe ratio analogous to implied volatility, we suppose that the options position is static, and the investment portfolio can only be changed by dynamically trading in the underlying asset.

Additionally, an investor can also compare the relative attractiveness of different European options portfolios using the implied Sharpe ratio. For this purpose, the price $p$ in (2) will be replaced by the price of the portfolio. For example, in the case of a risk reversal, the European option price $p$ will be replaced by the difference between the price of an out-of-the-money European call and an out-of-the-money European put. Comparison between other popular options strategies can also be made by appropriately choosing the price and payoff function of the European option used in Definition 3. Typically, an investor can only take certain options positions depending on the market access, risk guidelines and various other reasons. Thus, the implied Sharpe ratio acts as an essential tool to differentiate between different options portfolios for investment. 
As mentioned earlier, in the case of an exponential utility function, the implied Sharpe ratio will be independent of the starting wealth level, thus making it a better choice than directly observing the value function, which depends on the starting wealth. Investors with different wealth levels can just use the implied Sharpe ratio to make informed investment decisions instead of using the value function. Another possible alternative to compare the attractiveness of different European options could be their indifference price. In the context of an incomplete market, the indifference price per claim for $\nu$ European contingent claims is defined as the unique solution $u \equiv u(t, s, w, \nu)$ of the equation

$$
\mathrm{V}(t, s, w, 0)=\mathrm{V}(t, s, w-\nu u, \nu) .
$$

However, two European options with different implied Sharpe ratios can have the same indifference price. Therefore, the ranking of European options produced by the implied Sharpe ratios is more suitable than the one generated by using the indifference prices.

Next, we establish the theoretical result which ensures the existence and uniqueness of the implied Sharpe ratio.

Theorem 1. The implied Sharpe ratio defined in (2) exists and is unique.

Proof. The function $\mathrm{V}^{\mathrm{M}}(t, w ; \lambda)$ is the solution of the following partial differential equation:

$$
\partial_{t} \mathrm{~V}-\frac{1}{2} \lambda^{2} \frac{\left(\partial_{w} \mathrm{~V}\right)^{2}}{\partial_{w}^{2} \mathrm{~V}}=0, \quad \mathrm{~V}(\mathrm{~T}, w)=\mathrm{U}(w)
$$

It is also strictly increasing and strictly concave with respect to the state variable $w$. Thus, we have $\partial_{w}^{2} \mathrm{~V}^{\mathrm{M}}<0$. Next, suppose $\lambda>\lambda^{\prime}$, and let $\mathrm{V}^{\mathrm{M}}(t, w ; \lambda)$ and $\mathrm{V}^{\mathrm{M}}\left(t, w ; \lambda^{\prime}\right)$ denote the solutions to Equation (3) for $\lambda$ and $\lambda^{\prime}$, respectively. Then, we get

$$
\begin{aligned}
& \partial_{t} \mathrm{~V}^{\mathrm{M}}\left(t, w ; \lambda^{\prime}\right)-\frac{1}{2} \lambda^{2}\left(\frac{\partial_{w} \mathrm{~V}^{\mathrm{M}}}{\partial_{w}^{2} \mathrm{~V}^{\mathrm{M}}}\right)^{2}\left(t, w ; \lambda^{\prime}\right) \partial_{w}^{2} \mathrm{~V}^{\mathrm{M}}\left(t, w ; \lambda^{\prime}\right) \\
& =\partial_{t} \mathrm{~V}^{\mathrm{M}}\left(t, w ; \lambda^{\prime}\right)-\frac{1}{2} \lambda^{\prime}\left(\frac{\partial_{w} \mathrm{~V}^{\mathrm{M}}}{\partial_{w}^{2} \mathrm{~V}^{\mathrm{M}}}\right)^{2}\left(t, w ; \lambda^{\prime}\right) \partial_{w}^{2} \mathrm{~V}^{\mathrm{M}}\left(t, w ; \lambda^{\prime}\right) \\
& +\frac{1}{2}\left(\lambda^{\prime 2}-\lambda^{2}\right)\left(\frac{\partial_{w} \mathrm{~V}^{\mathrm{M}}}{\partial_{w}^{2} \mathrm{~V}^{\mathrm{M}}}\right)^{2}\left(t, w ; \lambda^{\prime}\right) \partial_{w}^{2} \mathrm{~V}^{\mathrm{M}}\left(t, w ; \lambda^{\prime}\right)>0
\end{aligned}
$$

Therefore, $\mathrm{V}^{\mathrm{M}}\left(t, w ; \lambda^{\prime}\right)$ is a subsolution to the equation solved by $\mathrm{V}^{\mathrm{M}}(t, w ; \lambda)$ with the terminal condition which is independent of $\lambda$ and $\mathrm{V}^{\mathrm{M}}\left(\mathrm{T}, w ; \lambda^{\prime}\right)=\mathrm{V}^{\mathrm{M}}(\mathrm{T}, w ; \lambda)$. Thus, we have that for $\lambda>\lambda^{\prime}$ and $t<\mathrm{T}, \mathrm{V}^{\mathrm{M}}(t, w ; \lambda)>$ $\mathrm{V}^{\mathrm{M}}\left(t, w ; \lambda^{\prime}\right)$. In other words, $\mathrm{V}^{\mathrm{M}}$ is strictly increasing in $\lambda$. Moreover, for any $\lambda, \mathrm{V}^{\mathrm{M}}(t, w ; \lambda) \geq \mathrm{U}(w)$ which guarantees the existence and uniqueness of the solution to (2).

In the next section, we study the implied Sharpe ratio in a class of incomplete market models that are Markov. In the absence of closed-form formulas of the implied Sharpe ratio, we use a Taylor series expansion technique for nolinear PDEs developed by Lorig (2018) to find semi-explicit approximation in terms of the risk-aversion parameter and other model parameters.

\section{$3 \quad$ Markov market setting}

To study the behaviour of the implied Sharpe ratio with respect to market parameters, we specialize to the setting of a Markov market model. We suppose that the dynamics of $\mathrm{S}$ are of the following form

$$
\begin{aligned}
\mathrm{S}_{t} & =\exp \left(\mathrm{X}_{t}\right) \\
\mathrm{dX}_{t} & =\left(\mu\left(\mathrm{X}_{t}, \mathrm{Y}_{t}\right)-\frac{1}{2} \sigma^{2}\left(\mathrm{X}_{t}, \mathrm{Y}_{t}\right)\right) \mathrm{d} t+\sigma\left(\mathrm{X}_{t}, \mathrm{Y}_{t}\right) \mathrm{dB}_{t}^{\mathrm{X}}
\end{aligned}
$$




$$
\mathrm{dY} \mathrm{Y}_{t}=c\left(\mathrm{X}_{t}, \mathrm{Y}_{t}\right) \mathrm{d} t+\beta\left(\mathrm{X}_{t}, \mathrm{Y}_{t}\right)\left(\rho \mathrm{dB}_{t}^{\mathrm{X}}+\sqrt{1-\rho^{2}} \mathrm{~dB}_{t}^{\mathrm{Y}}\right)
$$

where $\mathrm{B}^{\mathrm{X}}=\left(\mathrm{B}_{t}^{\mathrm{X}}\right)_{0 \leq t \leq \mathrm{T}}$ and $\mathrm{B}^{\mathrm{Y}}=\left(\mathrm{B}_{t}^{\mathrm{Y}}\right)_{0 \leq t \leq \mathrm{T}}$ are independent Brownian motions. We assume that the system of stochastic differential equations (4)-(5) admits a unique strong solution (X, Y) adapted to filtration $\mathbb{F}=\left(\mathcal{F}_{t}\right)_{0 \leq t \leq \mathrm{T}}$. Next, for $\pi_{t}$ units of currency invested in $\mathrm{S}$ at time $t$, the investor's wealth process $\mathrm{W}$ satisfies the following equation

$$
\mathrm{dW}_{t}=\frac{\pi_{t}}{\mathrm{~S}_{t}} \mathrm{~d} \mathrm{~S}_{t}=\pi_{t} \mu\left(\mathrm{X}_{t}, \mathrm{Y}_{t}\right) \mathrm{d} t+\pi_{t} \sigma\left(\mathrm{X}_{t}, \mathrm{Y}_{t}\right) \mathrm{dB}_{t}^{\mathrm{X}}
$$

The value function of an investor with $\nu$ European-style options and initial wealth level $w$ is given as

$$
\mathrm{V}(t, x, y, w, \nu)=\sup _{\pi \in \Pi} \mathbb{E}_{t, x, y, w}\left[\mathrm{U}\left(\mathrm{W}_{\mathrm{T}}+\nu \varphi\left(\mathrm{X}_{\mathrm{T}}\right)\right)\right] .
$$

Each European option with payoff function $\varphi$ and maturity $\mathrm{T}$ is assumed to have price $p$, which is obtained by computing the expectation of the option's payoff under the market's chosen pricing measure. We adopt the primal approach to solve for $\mathrm{V}$ and assume that the value function belongs to $C^{1,2,2,2}\left([0, T] \times \mathbb{R}^{3}\right)$. Under this assumption, by following the usual dynamic programming principle (see, for example, Chapter 3 Pham (2009)), V satisfies the Hamilton-Jacobi-Bellman (HJB) equation

$$
0=\left(\partial_{t}+\mathcal{A}\right) \mathrm{V}+\max _{\pi \in \mathrm{R}} \mathcal{A}^{\pi} \mathrm{V}, \quad \mathrm{V}(\mathrm{T}, x, y, w, \nu)=\mathrm{U}(w+\nu \varphi(x))
$$

where the operators $\mathcal{A}$ and $\mathcal{A}^{\pi}$ are given as

$$
\begin{aligned}
\mathcal{A}= & \left(\mu(x, y)-\frac{1}{2} \sigma^{2}(x, y)\right) \partial_{x}+\frac{1}{2} \sigma^{2}(x, y) \partial_{x}^{2}+c(x, y) \partial_{y}+\frac{1}{2} \beta^{2}(x, y) \partial_{y}^{2}+\rho \sigma(x, y) \beta(x, y) \partial_{x} \partial_{y} \\
\mathcal{A}^{\pi}= & \pi(t, x, y, w) \mu(x, y) \partial_{w}+\frac{1}{2} \pi^{2}(t, x, y, w) \sigma^{2}(x, y) \partial_{w}^{2}+\pi(t, x, y, w) \rho \sigma(x, y) \beta(x, y) \partial_{y} \partial_{w} \\
& +\pi(t, x, y, w) \sigma^{2}(x, y) \partial_{x} \partial_{w} .
\end{aligned}
$$

The candidate optimal strategy $\pi^{\star}$ is obtained by maximizing $\mathcal{A}^{\pi} \mathrm{V}$ which gives us

$$
\pi^{\star}=\underset{\pi \in \mathbb{R}}{\arg \max } \mathcal{A}^{\pi} \mathrm{V}=-\left(\frac{\mu\left(\partial_{w} \mathrm{~V}\right)+\rho \beta \sigma\left(\partial_{y} \partial_{w} \mathrm{~V}\right)+\sigma^{2}\left(\partial_{x} \partial_{w} \mathrm{~V}\right)}{\sigma^{2} \partial_{w}^{2} \mathrm{~V}}\right) .
$$

In the above formula, we have suppressed the arguments $(t, x, y, w, \nu)$ for simplicity of notation. We will do so from now on, wherever it causes no confusion, in order to keep the notation manageable.

Inserting the optimal strategy $\pi^{\star}$ in HJB equation (6) yields the following:

$$
0=\left(\partial_{t}+\mathcal{A}\right) \mathrm{V}+\mathcal{H}(\mathrm{V}), \quad \mathrm{V}(\mathrm{T}, x, y, w, \nu)=\mathrm{U}(w+\nu \varphi(x))
$$

where the Hamiltonian $\mathcal{H}(\mathrm{V})$ is a nonlinear term given as

$$
\begin{aligned}
\mathcal{H}(\mathrm{V})= & -\frac{1}{2} \lambda^{2} \frac{\left(\partial_{w} \mathrm{~V}\right)^{2}}{\partial_{w}^{2} \mathrm{~V}}-\rho \beta \lambda \frac{\left(\partial_{w} \mathrm{~V}\right)\left(\partial_{y} \partial_{w} \mathrm{~V}\right)}{\partial_{w}^{2} \mathrm{~V}}-\frac{1}{2} \rho^{2} \beta^{2} \frac{\left(\partial_{y} \partial_{w} \mathrm{~V}\right)^{2}}{\partial_{w}^{2} \mathrm{~V}} \\
& -\mu \frac{\left(\partial_{w} \mathrm{~V}\right)\left(\partial_{x} \partial_{w} \mathrm{~V}\right)}{\partial_{w}^{2} \mathrm{~V}}-\rho \sigma \beta \frac{\left(\partial_{x} \partial_{w} \mathrm{~V}\right)\left(\partial_{y} \partial_{w} \mathrm{~V}\right)}{\partial_{w}^{2} \mathrm{~V}}-\frac{1}{2} \sigma^{2} \frac{\left(\partial_{x} \partial_{w} \mathrm{~V}\right)^{2}}{\partial_{w}^{2} \mathrm{~V}}, \quad \lambda:=\frac{\mu}{\sigma} .
\end{aligned}
$$

Note that $\lambda$ defined above is the instantaneous Sharpe ratio of the risky asset $\mathrm{S}$. In the rest of the study, we fix the utility function $\mathrm{U}$ to be of the exponential form:

$$
\mathrm{U}(x)=-\frac{1}{\gamma} \mathrm{e}^{-\gamma x}, \quad \gamma>0,
$$

where $\gamma$ is the risk-aversion parameter. This choice of utility function allows us to obtain a form of the implied Sharpe ratio which is independent of the initial wealth level. The Merton value function in this case is given as

$$
\mathrm{V}^{\mathrm{M}}(t, w ; \lambda)=-\frac{1}{\gamma} \exp \left(-\gamma w-(\mathrm{T}-t) \frac{1}{2} \lambda^{2}\right) .
$$


One can verify by direct substitution that the above expression satisfies (3). Inspired by the form of the Merton value function for exponential utility, we make the following ansatz to solve the HJB equation (7):

$$
\mathrm{V}(t, x, y, w, \nu)=-\frac{1}{\gamma} \exp (-\gamma w+\psi(t, x, y, \nu)) .
$$

We find that the function $\psi$ satisfies the following equation

$$
0=\left(\partial_{t}+\widetilde{\mathcal{A}}\right) \psi+\mathcal{B}(\psi), \quad \psi(\mathrm{T}, x, y, \nu)=-\gamma \nu \varphi(x)
$$

where the linear operator $\widetilde{\mathcal{A}}$ and the nonlinear operator $\mathcal{B}$ are given by

$$
\widetilde{\mathcal{A}}=\frac{1}{2} \sigma^{2}\left(\partial_{x}^{2}-\partial_{x}\right)+(c-\rho \beta \lambda) \partial_{y}+\frac{1}{2} \beta^{2} \partial_{y}^{2}+\rho \sigma \beta \partial_{x} \partial_{y}, \quad \mathcal{B}(\psi)=\left(1-\rho^{2}\right)\left(\frac{1}{2} \beta^{2}\right)\left(\partial_{y} \psi\right)^{2}-\frac{1}{2} \lambda^{2} .
$$

To find a formula for the implied Sharpe ratio $\Lambda$, we solve the following equation

$$
\mathrm{V}^{\mathrm{M}}(t, w ; \Lambda)=\mathrm{V}(t, x, y, w-\nu p, \nu) .
$$

It can be checked that (9) is satisfied if and only if

$$
\gamma \nu p+\psi=-(\mathrm{T}-t) \frac{1}{2}(\Lambda)^{2}
$$

where, as a reminder, $p$ is the price of the European option with payoff $\varphi\left(\mathrm{X}_{\mathrm{T}}\right)$, as computed under the market's chosen pricing measure. As $p$ is independent of $\gamma$, we can obtain a relationship between the implied Sharpe ratio and investor's risk-aversion parameter by solving for $\psi$. In equation (8), we observe that $\psi$ depends on $\gamma$ only through the terminal condition. However, due to the presence of non-linearity $\mathcal{B}(\psi)$ in (8), deriving a closed-form formula for the relationship between $\psi$ and $\gamma$ is not possible. Even without the non-linearity in (8), the standard theory of partial differential equations (see Chapter 2, Section 4 Friedman (2008)) is not directly applicable to derive the relationship.

\section{Asymptotic approximation formulas}

In this section, we will use a Taylor series expansion method, as developed by Lorig (2018), to derive asymptotic approximations for $\psi$, the option price $p$ and the implied Sharpe ratio $\Lambda$.

\subsection{Asymptotic approximation formulas for $\psi$}

Let $\chi$ be any of the coefficient functions appearing in the operators $\widetilde{\mathcal{A}}$ or $\mathcal{B}(\cdot)$, that is

$$
\chi \in\left\{\left(\frac{1}{2} \sigma^{2}\right),(c-\rho \beta \lambda),(\rho \sigma \beta),\left(\frac{1}{2} \beta^{2}\right),\left(\frac{1}{2} \lambda^{2}\right)\right\} .
$$

Next, fix a point $(\bar{x}, \bar{y}) \in \mathbb{R}^{2}$ and define the following family of functions indexed by $\varepsilon \in[0,1]$ :

$$
\chi^{\varepsilon}(x, y):=\chi(\bar{x}+\varepsilon(x-\bar{x}), \bar{y}+\varepsilon(y-\bar{y})) .
$$

Observe that

$$
\left.\chi^{\varepsilon}(x, y)\right|_{\varepsilon=1}=\chi(x, y),\left.\quad \chi^{\varepsilon}(x, y)\right|_{\varepsilon=0}=\chi(\bar{x}, \bar{y}) .
$$

Suppose the functions are analytic in a neighbourhood of $(\bar{x}, \bar{y})$ so that we have

$$
\chi^{\varepsilon}(x, y):=\sum_{n=0}^{\infty} \varepsilon^{n} \chi_{n}(x, y),
$$




$$
\chi_{n}(x, y):=\sum_{k=0}^{n} \chi_{n-k, k} \cdot(x-\bar{x})^{n-k}(y-\bar{y})^{k}, \quad \chi_{n-k, k}:=\frac{1}{(n-k) ! k !} \partial_{x}^{n-k} \partial_{y}^{k} \chi(\bar{x}, \bar{y}) .
$$

For the final $m$-th order approximation formula, we will only need the functions to be $m$-times differentiable. However, it will simplify the presentation if, for now, we assume the stronger condition of analyticity. Using the coefficient functions indexed by $\varepsilon$, consider the following family of PDEs indexed by $\varepsilon$ :

$$
0=\left(\partial_{t}+\tilde{\mathcal{A}}^{\varepsilon}\right) \psi^{\varepsilon}+\mathcal{B}^{\varepsilon}\left(\psi^{\varepsilon}\right), \quad \quad \psi^{\varepsilon}(\mathrm{T}, x, y, \nu)=-\gamma \nu \varphi(x),
$$

where $\widetilde{\mathcal{A}}^{\varepsilon}$ and $\mathcal{B}^{\varepsilon}(\cdot)$ are obtained from $\widetilde{\mathcal{A}}$ and $\mathcal{B}(\cdot)$ and by replacing the coefficients in these operators with their $\varepsilon$-counterparts defined in (11)

$$
\begin{aligned}
\widetilde{\mathcal{A}}^{\varepsilon} & =\left(\frac{1}{2} \sigma^{2}\right)^{\varepsilon}\left(\partial_{x}^{2}-\partial_{x}\right)+(c-\rho \beta \lambda)^{\varepsilon} \partial_{y}+\left(\frac{1}{2} \beta^{2}\right)^{\varepsilon} \partial_{y}^{2}+(\rho \sigma \beta)^{\varepsilon} \partial_{x} \partial_{y}, \\
\mathcal{B}^{\varepsilon}\left(\psi^{\varepsilon}\right) & =\left(1-\rho^{2}\right)\left(\frac{1}{2} \beta^{2}\right)^{\varepsilon}\left(\partial_{y} \psi^{\varepsilon}\right)^{2}-\left(\frac{1}{2} \lambda^{2}\right)^{\varepsilon} .
\end{aligned}
$$

Next, we suppose that $\psi^{\varepsilon}$ is given in powers of $\varepsilon$ as

$$
\psi^{\varepsilon}=\sum_{i=0}^{\infty} \varepsilon^{i} \psi_{i}
$$

Once we are able to solve for each $\psi_{i}$, the asymptotic approximation of $\psi$ is obtained by setting $\varepsilon=1$ in (13). To obtain the respective order terms $\psi_{i}$, we insert the expansion of $\psi^{\varepsilon}$ into (12) and collect the terms of like powers of $\varepsilon$. At the lowest order of $\varepsilon$, we have the following

$$
\mathcal{O}(1): \quad 0=\left(\partial_{t}+\widetilde{\mathcal{A}}_{0}\right) \psi_{0}+\left(1-\rho^{2}\right)\left(\frac{1}{2} \beta^{2}\right)_{0}\left(\partial_{y} \psi_{0}\right)^{2}-\left(\frac{1}{2} \lambda^{2}\right)_{0}, \quad \psi_{0}(\mathrm{~T}, x, y, \nu)=-\gamma \nu \varphi(x),
$$

where we have defined

$$
\widetilde{\mathcal{A}}_{n}:=\left(\frac{1}{2} \sigma^{2}\right)_{n}\left(\partial_{x}^{2}-\partial_{x}\right)+(c-\rho \beta \lambda)_{n} \partial_{y}+\left(\frac{1}{2} \beta^{2}\right)_{n} \partial_{y}^{2}+(\rho \sigma \beta)_{n} \partial_{x} \partial_{y}, \quad n \in\{0\} \cup \mathbb{N} .
$$

In the above, $\widetilde{\mathcal{A}}_{0}$ is a constant coefficient differential operator since we have that $\chi_{0}:=\chi(\bar{x}, \bar{y})$. It can be seen that $\psi_{0}$ is only a function of $(t, x)$. As such the equation becomes

$$
\mathcal{O}(1): \quad 0=\left(\partial_{t}+\widetilde{\mathcal{A}}_{0}\right) \psi_{0}-\left(\frac{1}{2} \lambda^{2}\right)_{0}, \quad \psi_{0}(\mathrm{~T}, x, \nu)=-\gamma \nu \varphi(x) .
$$

The higher order terms $m \geq 1$ are given as

$$
\mathcal{O}\left(\varepsilon^{m}\right): \quad \quad 0=\left(\partial_{t}+\widetilde{\mathcal{A}}_{0}\right) \psi_{m}+\mathrm{H}_{m}, \quad \psi_{m}(\mathrm{~T}, x, y, \nu)=0,
$$

where the source terms $\mathrm{H}_{m}$ are given as

$$
\begin{aligned}
\mathcal{O}\left(\varepsilon^{m}\right): & \mathrm{H}_{m}=\sum_{k=1}^{m} \widetilde{\mathcal{A}}_{k} \psi_{m-k}-\left(\frac{1}{2} \lambda^{2}\right)_{m}+\left(1-\rho^{2}\right) \sum_{k, i, j \in \mathrm{K}_{m}}\left(\frac{1}{2} \beta^{2}\right)_{k}\left(\partial_{y} \psi_{i}\right)\left(\partial_{y} \psi_{j}\right), \\
\mathrm{K}_{m} & =\left\{(i, k, j) \in \mathbb{N}_{0}^{3}: i+j+k=m, \text { and } i, j, k \neq m\right\} .
\end{aligned}
$$

In particular, the first and second order source terms are given by

$$
\begin{aligned}
\mathcal{O}(\varepsilon): & \mathrm{H}_{1}=\widetilde{\mathcal{A}}_{1} \psi_{0}-\left(\frac{1}{2} \lambda^{2}\right)_{1} \\
\mathcal{O}\left(\varepsilon^{2}\right): & \mathrm{H}_{2}=\widetilde{\mathcal{A}}_{2} \psi_{0}+\widetilde{\mathcal{A}}_{1} \psi_{1}-\left(\frac{1}{2} \lambda^{2}\right)_{2}+\left(1-\rho^{2}\right)\left(\frac{1}{2} \beta^{2}\right)_{0}\left(\partial_{y} \psi_{1}\right)^{2} .
\end{aligned}
$$

Before, we proceed to solve for terms $\psi_{i}$, we state a few fundamental results which will be useful for our calculations. The constant coefficient elliptic operator $\widetilde{\mathcal{A}}_{0}$ in $(15)$, gives rise to the semigroup $\widetilde{\mathcal{P}}_{0}\left(t, t_{1}\right)$ defined by

$$
\widetilde{\mathcal{P}}_{0}\left(t, t_{1}\right) \eta(x, y):=\int_{\mathbb{R}^{2}} \mathrm{~d} x \mathrm{~d} y \widetilde{\Gamma}_{0}\left(t, x, y ; t_{1}, x_{1}, y_{1}\right) \eta\left(x_{1}, y_{1}\right)
$$


where $\eta: \mathbb{R}^{2} \rightarrow \mathbb{R}^{2}$ is a generic test function and $\widetilde{\Gamma}_{0}$ is the fundamental solution to the linear operator $\left(\partial_{t}+\widetilde{\mathcal{A}}_{0}\right)$ given as

$$
\widetilde{\Gamma}_{0}\left(t, x, y ; t_{1}, x_{1}, y_{1}\right)=\frac{1}{\sqrt{(2 \pi)^{2}|\widetilde{\mathbf{C}}|}} \exp \left(-\frac{1}{2} \widetilde{\mathbf{m}}^{\top} \widetilde{\mathbf{C}}^{-1} \widetilde{\mathbf{m}}\right)
$$

The covariance matrix $\widetilde{\mathbf{C}}$ and vector $\widetilde{\mathbf{m}}$ are given as

$$
\widetilde{\mathbf{C}}=\left(t_{1}-t\right)\left(\begin{array}{cc}
\left(\sigma^{2}\right)_{0} & (\rho \sigma \beta)_{0} \\
(\rho \sigma \beta)_{0} & \left(\beta^{2}\right)_{0}
\end{array}\right), \quad \widetilde{\mathbf{m}}=\left(t_{1}-t\right)\left(\begin{array}{c}
x_{1}-x-\left(t_{1}-t\right)\left(-\frac{1}{2} \sigma^{2}\right)_{0} \\
y_{1}-y-\left(t_{1}-t\right)(c-\rho \beta \lambda)_{0}
\end{array}\right)
$$

To obtain the terms in the expansion of $\psi^{\varepsilon}$, it will also be helpful to introduce the following operators

$$
\begin{array}{ll}
\widetilde{X}\left(t, t_{1}\right):=x+\left(t_{1}-t\right)\left(-\left(\frac{1}{2} \sigma^{2}\right)_{0}+2\left(\frac{1}{2} \sigma^{2}\right)_{0} \partial_{x}+(\rho \sigma \beta)_{0} \partial_{y}\right), & t_{1} \geq t, \\
\widetilde{y}\left(t, t_{1}\right):=y+\left(t_{1}-t\right)\left((c-\rho \beta \lambda)_{0}+2\left(\frac{1}{2} \beta^{2}\right)_{0} \partial_{y}+(\rho \sigma \beta)_{0} \partial_{x}\right), & t_{1} \geq t \\
\widetilde{\mathcal{S}}_{n}\left(t, t_{1}\right):=\widetilde{\mathcal{A}}_{n}\left(\widetilde{X}\left(t, t_{1}\right), \widetilde{y}\left(t, t_{1}\right)\right), & t_{1} \geq t, n \geq 1,
\end{array}
$$

where the notation $\widetilde{\mathcal{A}}_{n}\left(\widetilde{X}\left(t, t_{1}\right), \widetilde{y}\left(t, t_{1}\right)\right)$ indicates that the $(x, y)$-dependence in coefficients $\widetilde{\mathcal{A}}_{n} \equiv \widetilde{\mathcal{A}}_{n}(x, y)$, has been replaced with $\left(\widetilde{X}\left(t, t_{1}\right), \widetilde{y}\left(t, t_{1}\right)\right)$. For example, in $\widetilde{\mathcal{G}}_{1}\left(t, t_{1}\right)=\widetilde{\mathcal{A}}_{1}\left(\widetilde{X}\left(t, t_{1}\right), \widetilde{y}\left(t, t_{1}\right)\right)$, the term

$$
\left(\frac{1}{2} \sigma^{2}\right)_{1}\left(\partial_{x}^{2}-\partial_{x}\right)=\left(\left(\frac{1}{2} \sigma^{2}\right)_{1,0}(x-\bar{x})+\left(\frac{1}{2} \sigma^{2}\right)_{0,1}(y-\bar{y})\right)\left(\partial_{x}^{2}-\partial_{x}\right)
$$

becomes

$$
\left(\left(\frac{1}{2} \sigma^{2}\right)_{1,0}\left(\widetilde{x}\left(t, t_{1}\right)-\bar{x}\right)+\left(\frac{1}{2} \sigma^{2}\right)_{0,1}\left(\widetilde{y}\left(t, t_{1}\right)-\bar{y}\right)\right)\left(\partial_{x}^{2}-\partial_{x}\right) .
$$

It is assumed above and throughout the following computations that if an operator is followed by nothing, it acts on the constant 1. To obtain semi-explicit approximation formulas, we fix the European option payoff function as a call option, that is, $\varphi(x)=\left(\mathrm{e}^{x}-\mathrm{e}^{k}\right)^{+}$where $k$ is the $\log$ strike. Using this choice of $\varphi$, we obtain the second order approximation of $\psi$ in (13) using the following result:

Proposition 2. Let $\psi_{0}$ be the unique classical solution of (15) and $\psi_{1}$ and $\psi_{2}$ be the unique classical solutions of (16) with source terms $\mathrm{H}_{1}$ (17) and $\mathrm{H}_{2}$ (18), respectively. Assume that the coefficients $\left(\frac{1}{2} \lambda^{2}\right),\left(\frac{1}{2} \sigma^{2}\right),(c-$ $\rho \beta \lambda),\left(\frac{1}{2} \beta^{2}\right)$ and $(\rho \sigma \beta)$ belong to the class of $\mathrm{C}^{2}\left(\mathbb{R}^{2}\right)$ functions. Then, omitting arguments $(x, y)$ for clarity, we have

$$
\begin{aligned}
\psi_{0}(t)= & -\gamma \nu p^{\mathrm{BS}}(t)-\left(\frac{1}{2} \lambda^{2}\right)_{0}(\mathrm{~T}-t), \\
p^{\mathrm{BS}}(t):= & \mathrm{e}^{x} \Phi\left(d_{+}(t)\right)-\mathrm{e}^{k} \Phi(d-(t)), \quad d_{ \pm}(t)=\frac{1}{\sigma_{0} \sqrt{\mathrm{T}-t}}\left(x-k \pm \frac{1}{2} \sigma_{0}^{2}(\mathrm{~T}-t)\right), \\
\psi_{1}(t)= & -\gamma \nu \int_{t}^{\mathrm{T}} \mathrm{d} t_{1} \widetilde{\mathcal{G}}_{1}\left(t, t_{1}\right) p^{\mathrm{BS}}(t)-\int_{t}^{\mathrm{T}} \mathrm{d} t_{1}\left(\frac{1}{2} \lambda^{2}\right)_{1}\left(\widetilde{\mathcal{X}}\left(t, t_{1}\right), \widetilde{y}\left(t, t_{1}\right)\right), \\
\psi_{2}(t)= & -\gamma \nu\left(\int_{t}^{\mathrm{T}} \mathrm{d} t_{1} \int_{t_{1}}^{\mathrm{T}} \mathrm{d} t_{2} \widetilde{\mathfrak{G}}_{1}\left(t, t_{1}\right) \widetilde{\mathcal{G}}_{1}\left(t_{1}, t_{2}\right)+\int_{t}^{\mathrm{T}} \mathrm{d} t_{1} \widetilde{\mathfrak{G}}_{2}\left(t, t_{1}\right)\right) p^{\mathrm{BS}}(t) \\
& -\int_{t}^{\mathrm{T}} \mathrm{d} t_{1}\left(\frac{1}{2} \lambda^{2}\right)_{2}\left(\widetilde{X}\left(t, t_{1}\right), \widetilde{y}\left(t, t_{1}\right)\right)-\int_{t}^{\mathrm{T}} \mathrm{d} t_{1} \widetilde{\mathfrak{G}}_{1}\left(t, t_{1}\right) \int_{t_{1}}^{\mathrm{T}} \mathrm{d} t_{2}\left(\frac{1}{2} \lambda^{2}\right)_{1}\left(\widetilde{X}\left(t, t_{2}\right), \widetilde{y}\left(t, t_{2}\right)\right) \\
& +\left(1-\rho^{2}\right)\left(\frac{1}{2} \beta^{2}\right)_{0}\left[\left(\frac{1}{2} \lambda^{2}\right)_{0,1}^{2} \frac{1}{3}(\mathrm{~T}-t)^{3}+2 \gamma \nu \int_{t}^{\mathrm{T}} \mathrm{d} t_{1}\left(\frac{1}{2} \lambda^{2}\right)_{0,1}\left(\mathrm{~T}-t_{1}\right) \partial_{y}\left(\int_{t_{1}}^{\mathrm{T}} \mathrm{d} t_{2} \widetilde{\mathcal{G}}_{1}\left(t, t_{2}\right)\right) p^{\mathrm{BS}}(t)\right. \\
& \left.+\gamma^{2} \nu^{2} \frac{\left(\frac{1}{2} \sigma^{2}\right)_{0,1}^{2}}{2 \pi \sigma_{0}^{2}} \int_{t}^{\mathrm{T}} \mathrm{d} t_{1} \frac{\left(\mathrm{T}-t_{1}\right)^{3 / 2}}{\sqrt{\mathrm{T}-t+t_{1}-t}} \exp \left(2 k-\frac{\left.\left.\left((k-x)+\frac{1}{2} \sigma_{0}^{2}(\mathrm{~T}-t)\right)^{2}\right)\right] .}{\sigma_{0}^{2}\left(\mathrm{~T}-t+t_{1}-t\right)}\right)\right]
\end{aligned}
$$


Proof. See Appendix B.

To study the effect of the risk-aversion parameter $\gamma$ on the implied Sharpe ratio $\Lambda$, we derive the asymptotic approximation formula only up to the second order. The second order approximation is sufficient to perform a comparative-static study with respect to the risk-aversion parameter and to compare the relative attractiveness of the available options in the market. However, to compare the accuracy of our second order approximation formulas, we conduct a comparison with the numerical solutions obtained via finite-difference methods of PDEs of type (8) in Section 5.4.

\subsection{Asymptotic approximation formulas for $p$}

In an incomplete market, the investors may not agree on their choice of the pricing measure. Thus, price $p$ of the European option with payoff $\varphi$ will depend on the chosen model. In our study, as we fix the model for our reference investor in (4)-(5), it makes sense to obtain $p$ under the appropriate pricing measure based on the model instead of using a market-given European option price.

We suppose that the pricing measure $\widehat{\mathbb{P}}$ is related to the physical measure $\mathbb{P}$ through the following RadonNikodym derivative:

$$
\frac{\mathrm{d} \widehat{\mathbb{P}}}{\mathrm{dP}}=\exp \left(-\frac{1}{2} \int_{0}^{\mathrm{T}}\left(\frac{\mu^{2}\left(\mathrm{X}_{t}, \mathrm{Y}_{t}\right)}{\sigma^{2}\left(\mathrm{X}_{t}, \mathrm{Y}_{t}\right)}+\Omega^{2}\left(\mathrm{X}_{t}, \mathrm{Y}_{t}\right)\right) \mathrm{d} t-\int_{0}^{\mathrm{T}} \frac{\mu\left(\mathrm{X}_{t}, \mathrm{Y}_{t}\right)}{\sigma\left(\mathrm{X}_{t}, \mathrm{Y}_{t}\right)} \mathrm{dB}_{t}^{\mathrm{X}}-\int_{0}^{\mathrm{T}} \Omega\left(\mathrm{X}_{t}, \mathrm{Y}_{t}\right) \mathrm{dB} B_{t}^{\mathrm{Y}}\right)
$$

Defining $(\widehat{\mathbb{P}}, \mathbb{F})$-Brownian motions $\widehat{\mathrm{B}}^{\mathrm{X}}$ and $\widehat{\mathrm{B}}^{\mathrm{Y}}$ by

$$
\widehat{\mathrm{B}}^{\mathrm{X}}:=\mathrm{B}_{t}^{\mathrm{X}}+\int_{0}^{t} \frac{\mu\left(\mathrm{X}_{s}, \mathrm{Y}_{s}\right)}{\sigma\left(\mathrm{X}_{s}, \mathrm{Y}_{s}\right)} \mathrm{d} s, \quad \widehat{\mathrm{B}}^{\mathrm{Y}}:=\mathrm{B}_{t}^{\mathrm{Y}}+\int_{0}^{t} \Omega\left(\mathrm{X}_{s}, \mathrm{Y}_{s}\right) \mathrm{d} s,
$$

we see that the dynamics of $(\mathrm{X}, \mathrm{Y})$ can be written under $\widehat{\mathbb{P}}$ as

$$
\begin{aligned}
& \mathrm{dX} \mathrm{X}_{t}=-\frac{1}{2} \sigma^{2}\left(\mathrm{X}_{t}, \mathrm{Y}_{t}\right) \mathrm{d} t+\sigma\left(\mathrm{X}_{t}, \mathrm{Y}_{t}\right) \mathrm{d} \widehat{\mathrm{B}}_{t}^{\mathrm{X}}, \\
& \mathrm{d} \mathrm{Y}_{t}=b\left(\mathrm{X}_{t}, \mathrm{Y}_{t}\right) \mathrm{d} t+\beta\left(\mathrm{X}_{t}, \mathrm{Y}_{t}\right)\left(\rho \mathrm{d} \widehat{\mathrm{B}}_{t}^{\mathrm{X}}+\sqrt{1-\rho^{2}} \mathrm{~d} \widehat{\mathrm{B}}_{t}^{\mathrm{Y}}\right),
\end{aligned}
$$

where the function $b$ is given as

$$
b(x, y):=c(x, y)-\rho \beta(x, y) \lambda(x, y)-\sqrt{1-\rho^{2}} \beta(x, y) \Omega(x, y) .
$$

The price of a European-style option with payoff function $\varphi$, with $(\mathrm{X}, \mathrm{Y})$ having risk-neutral dynamics under $\widehat{\mathbb{P}}$, is given as

$$
p(t, x, y)=\widehat{\mathbb{E}}_{t, x, y} \varphi\left(\mathrm{X}_{\mathrm{T}}\right),
$$

where $\widehat{\mathbb{E}}$ denotes the expectation operator under $\widehat{\mathbb{P}}$ defined in (23). The function $p$ satisfies the linear pricing $\mathrm{PDE}$

$$
0=\left(\partial_{t}+\widehat{\mathcal{A}}\right) p, \quad p(\mathrm{~T}, x, y)=\varphi(x),
$$

where $\widehat{\mathcal{A}}:=\widetilde{\mathcal{A}}-\sqrt{1-\rho^{2}} \beta \Omega \partial_{y}$. To obtain asymptotic approximation formulas, once again we seek a solution $p^{\varepsilon}=\sum_{k=0}^{\infty} \varepsilon^{k} p_{k}$ to the following family of PDEs

$$
0=\left(\partial_{t}+\widehat{\mathcal{A}}^{\varepsilon}\right) p^{\varepsilon}, \quad p^{\varepsilon}(\mathrm{T}, x, y)=\varphi(x),
$$

where $\widehat{\mathcal{A}}^{\varepsilon}$ is the $\varepsilon$-counterpart of $\widehat{\mathcal{A}}$ obtained by replacing the coefficients in it with their $\varepsilon$-counterparts. Upon collecting the terms of like order of $\varepsilon$, we find that the individual terms satisfy

$$
\mathcal{O}(1): \quad \quad 0=\left(\partial_{t}+\widehat{\mathcal{A}}_{0}\right) p_{0}, \quad p_{0}(\mathrm{~T}, x)=\varphi(x)
$$




$$
\mathcal{O}\left(\varepsilon^{m}\right): \quad \quad 0=\left(\partial_{t}+\widehat{\mathcal{A}}_{0}\right) p_{m}+\mathrm{P}_{m}, \quad p_{m}(\mathrm{~T}, x, y)=0, m \geq 1
$$

where the $m$ th-order source term $\mathrm{P}_{m}$ is given by

$$
\mathcal{O}\left(\varepsilon^{m}\right): \quad \mathrm{P}_{m}=\sum_{k=1}^{m} \widehat{\mathcal{A}}_{k} p_{m-k}
$$

and $\widehat{\mathcal{A}}_{n}$ is given by

$$
\widehat{\mathcal{A}}_{n}:=\left(\frac{1}{2} \sigma^{2}\right)_{n}\left(\partial_{x}^{2}-\partial_{x}\right)+\left(c-\rho \beta \lambda-\sqrt{1-\rho^{2}} \beta \Omega\right)_{n} \partial_{y}+\left(\frac{1}{2} \beta^{2}\right)_{n} \partial_{y}^{2}+(\rho \sigma \beta)_{n} \partial_{x} \partial_{y}, \quad n \in\{0\} \cup \mathbb{N} .
$$

Like $\widetilde{\mathcal{A}}_{0}$ in Section $4.1, \widehat{\mathcal{A}}_{0}$ gives rise to a semigroup $\widehat{\mathcal{P}}_{0}\left(t, t_{1}\right)$ defined by

$$
\widehat{\mathcal{P}}_{0}\left(t, t_{1}\right) \eta(x, y):=\int_{\mathbb{R}^{2}} \mathrm{~d} x \mathrm{~d} y \widehat{\Gamma}_{0}\left(t, x, y ; t_{1}, x_{1}, y_{1}\right) \eta\left(x_{1}, y_{1}\right),
$$

where $\eta: \mathbb{R}^{2} \rightarrow \mathbb{R}^{2}$ is a generic test function and $\widehat{\Gamma}_{0}$ is the fundamental solution to the linear operator $\left(\partial_{t}+\widehat{\mathcal{A}}_{0}\right)$ given as

$$
\widehat{\Gamma}_{0}\left(t, x, y ; t_{1}, x_{1}, y_{1}\right)=\frac{1}{\sqrt{(2 \pi)^{2}|\widehat{\mathbf{C}}|}} \exp \left(-\frac{1}{2} \widehat{\mathbf{m}}^{\top} \widehat{\mathbf{C}}^{-1} \widehat{\mathbf{m}}\right)
$$

The covariance matrix $\widehat{\mathbf{C}}$ and vector $\widehat{\mathbf{m}}$ are given as

$$
\widehat{\mathbf{C}}=\left(t_{1}-t\right)\left(\begin{array}{cc}
\left(\sigma^{2}\right)_{0} & (\rho \sigma \beta)_{0} \\
(\rho \sigma \beta)_{0} & \left(\beta^{2}\right)_{0}
\end{array}\right), \quad \widehat{\mathbf{m}}=\left(t_{1}-t\right)\left(\begin{array}{c}
x_{1}-x-\left(t_{1}-t\right)\left(-\frac{1}{2} \sigma^{2}\right)_{0} \\
y_{1}-y-\left(t_{1}-t\right)\left(c-\rho \beta \lambda-\sqrt{1-\rho^{2}} \beta \Omega\right)_{0}
\end{array}\right) .
$$

Analogous to $\widetilde{x}, \widetilde{y}$ and $\widetilde{\mathcal{G}}$ in Section 4.1, we define $\widehat{x}, \widehat{y}$ and $\widehat{\mathcal{G}}$ as follows:

$$
\begin{array}{ll}
\widehat{X}\left(t, t_{1}\right):=x+\left(t_{1}-t\right)\left(-\left(\frac{1}{2} \sigma^{2}\right)_{0}+2\left(\frac{1}{2} \sigma^{2}\right)_{0} \partial_{x}+(\rho \sigma \beta)_{0} \partial_{y}\right), & t_{1} \geq t, \\
\widehat{y}\left(t, t_{1}\right):=y+\left(t_{1}-t\right)\left((c-\rho \beta \lambda)_{0}+2\left(\frac{1}{2} \beta^{2}\right)_{0} \partial_{y}+(\rho \sigma \beta)_{0} \partial_{x}\right), & t_{1} \geq t, \\
\widehat{\mathcal{G}}_{n}\left(t, t_{1}\right):=\widehat{\mathcal{A}}_{n}\left(\widehat{X}\left(t, t_{1}\right), \widehat{y}\left(t, t_{1}\right)\right), & t_{1} \geq t, n \geq 1 .
\end{array}
$$

The operators $\widehat{\mathcal{P}}_{0}, \widehat{x}, \widehat{y}$ and $\widehat{\mathcal{G}}$ satisfy the same properties as $\widetilde{\mathcal{P}}_{0}, \widetilde{x}, \widetilde{y}$ and $\widetilde{\mathcal{G}}$, respectively, in Section 4.1 . We obtain the following result for approximation of the European option price $p$ :

Proposition 3. Let $p_{0}$ be the unique classical solution of (25), and, $p_{1}$ and $p_{2}$ be the unique classical solutions of (26) with source terms $\mathrm{P}_{1}$ and $\mathrm{P}_{2}$, respectively, obtained from (27). Assume that the coefficients $\left(\frac{1}{2} \lambda^{2}\right),\left(\frac{1}{2} \sigma^{2}\right),\left(c-\rho \beta \lambda-\sqrt{1-\rho^{2}} \beta \Omega\right),\left(\frac{1}{2} \beta^{2}\right)$ and $(\rho \sigma \beta)$ belong to the class of $\mathrm{C}^{2}\left(\mathbb{R}^{2}\right)$ functions. Then, omitting arguments $(x, y)$ for clarity, we have

$$
\begin{aligned}
p_{0}(t) & =p^{\mathrm{BS}}(t), \\
p^{\mathrm{BS}}(t) & :=\mathrm{e}^{x} \Phi\left(d_{+}(t)\right)-\mathrm{e}^{k} \Phi\left(d_{-}(t)\right), \quad d_{ \pm}(t)=\frac{1}{\sigma_{0} \sqrt{\mathrm{T}-t}}\left(x-k \pm \frac{1}{2} \sigma_{0}^{2}(\mathrm{~T}-t)\right), \\
p_{1}(t) & =\int_{t}^{\mathrm{T}} \mathrm{d} t_{1} \widehat{\mathcal{G}}_{1}\left(t, t_{1}\right) p^{\mathrm{BS}}(t), \\
p_{2}(t) & =\left(\int_{t}^{\mathrm{T}} \mathrm{d} t_{1} \widehat{\mathcal{G}}_{2}\left(t, t_{1}\right)+\int_{t}^{\mathrm{T}} \mathrm{d} t_{1} \int_{t_{1}}^{\mathrm{T}} \mathrm{d} t_{2} \widehat{\mathcal{G}}_{1}\left(t, t_{1}\right) \widehat{\mathcal{G}}_{1}\left(t_{1}, t_{2}\right)\right) p^{\mathrm{BS}}(t) .
\end{aligned}
$$

Proof. See Appendix B. 


\subsection{Asymptotic approximation formulas for $\Lambda$}

We use the results in Proposition 2 and 3 to obtain an asymptotic formula for the implied Sharpe ratio $\Lambda$. Let us define $\Lambda^{\varepsilon}$ is the positive solution of the following equation

$$
\gamma \nu p^{\varepsilon}+\psi^{\varepsilon}=-(\mathrm{T}-t) \frac{1}{2}\left(\Lambda^{\varepsilon}\right)^{2},
$$

which is obtained by replacing $p$ and $\psi$ in (10) by their $\varepsilon$ counterparts. Recalling that $\left.p^{\varepsilon}\right|_{\varepsilon=1}=p$ and $\left.\psi^{\varepsilon}\right|_{\varepsilon=1}=\psi$, it follows that $\left.\Lambda^{\varepsilon}\right|_{\varepsilon=1}=\Lambda$. Thus, in order to find an asymptotic approximation for $\Lambda$, we expand $\Lambda^{\varepsilon}$ in powers of $\varepsilon$ as follows

$$
\Lambda^{\varepsilon}=\sum_{i=0}^{\infty} \varepsilon^{i} \Lambda_{i}
$$

Once we obtain expressions for $\left(\Lambda_{i}\right)$, our $n$-th order approximation for $\Lambda$ will be obtained by truncating the above series at order $n$ and setting $\varepsilon=1$. In order to find explicit expressions for the terms $\left(\Lambda_{i}\right)$ we insert the expansions for $p^{\varepsilon}, \psi^{\varepsilon}$ and $\Lambda^{\varepsilon}$ into (30) and collect terms of like order in $\varepsilon$. We obtain

$$
\begin{array}{ll}
\mathcal{O}(1): & \gamma \nu p_{0}+\psi_{0}=-(\mathrm{T}-t) \frac{1}{2} \Lambda_{0}^{2} \\
\mathcal{O}\left(\varepsilon^{k}\right): & \gamma \nu p_{k}+\psi_{k}=-(\mathrm{T}-t) \frac{1}{2}\left(2 \Lambda_{0} \Lambda_{k}+\sum_{i+j=k} \Lambda_{i} \Lambda_{j}\right),
\end{array} \quad k \geq 1 .
$$

By solving for $\Lambda_{k}$, we obtain

$$
\begin{aligned}
\mathcal{O}(1): & \Lambda_{0} & =\sqrt{\frac{\gamma \nu p_{0}+\psi_{0}}{-\frac{1}{2}(\mathrm{~T}-t)}} \\
\mathcal{O}\left(\varepsilon^{k}\right): & \Lambda_{k} & =-\frac{1}{(\mathrm{~T}-t) \Lambda_{0}}\left(\gamma \nu p_{k}+\psi_{k}+(\mathrm{T}-t) \frac{1}{2} \sum_{i+j=k} \Lambda_{i} \Lambda_{j}\right) .
\end{aligned}
$$

Proposition 4. Let $p_{0}$ be the unique classical solution of (25) and $p_{1}$ and $p_{2}$ be the unique classical solutions of (26) with source terms $\mathrm{P}_{1}$ and $\mathrm{P}_{2}$, respectively, obtained from (27). Furthermore, let $\psi_{0}$ be the unique classical solution of (15) and $\psi_{1}$ and $\psi_{2}$ be the unique classical solutions of (16) with source terms $\mathrm{H}_{1}$ (17) and $\mathrm{H}_{2}(18)$, respectively. Assume that the coefficients $\left(\frac{1}{2} \lambda^{2}\right),\left(\frac{1}{2} \sigma^{2}\right),(c-\rho \beta \lambda),\left(c-\rho \beta \lambda-\sqrt{1-\rho^{2}} \beta \Omega\right),\left(\frac{1}{2} \beta^{2}\right)$ and $(\rho \sigma \beta)$ belong to the class of $\mathrm{C}^{2}\left(\mathbb{R}^{2}\right)$ functions. Then, the second order approximation of the implied Sharpe ratio, defined as

$$
\bar{\Lambda}_{2}(t, x, y, w, \nu):=\left(\Lambda_{0}+\Lambda_{1}+\Lambda_{2}\right)(t, x, y, w, \nu)
$$

is given explicitly by

$$
\begin{aligned}
& \Lambda_{0}=\lambda_{0} \\
& \Lambda_{1}=-\frac{1}{2 \Lambda_{0}(\mathrm{~T}-t)}\left(\gamma \nu p_{1}+\psi_{1}\right), \\
& \Lambda_{2}=-\frac{1}{2 \Lambda_{0}(\mathrm{~T}-t)}\left(\gamma \nu p_{2}+\psi_{2}+(\mathrm{T}-t) \frac{\Lambda_{1}^{2}}{2}\right) .
\end{aligned}
$$

The above result provides an approximate relationship between $\Lambda \approx \bar{\Lambda}_{2}$, the risk-aversion parameter $\gamma$ and the instantaneous Sharpe ratio $\lambda$.

Remark 1. If we set $\Omega \equiv 0$ in (23), the pricing measure $\widehat{\mathbb{P}}$ corresponds to the minimal martingale measure $\widetilde{\mathbb{P}}$ as defined in Follmer and Schweizer (1991)

$$
\frac{\mathrm{d} \widetilde{\mathbb{P}}}{\mathrm{dP}}:=\exp \left(-\frac{1}{2} \int_{0}^{\mathrm{T}} \frac{\mu^{2}\left(\mathrm{X}_{t}, \mathrm{Y}_{t}\right)}{\sigma^{2}\left(\mathrm{X}_{t}, \mathrm{Y}_{t}\right)} \mathrm{d} t-\int_{0}^{\mathrm{T}} \frac{\mu\left(\mathrm{X}_{t}, \mathrm{Y}_{t}\right)}{\sigma\left(\mathrm{X}_{t}, \mathrm{Y}_{t}\right)} \mathrm{dB}_{t}^{\mathrm{X}}\right)
$$


Moreover, if the market's chosen pricing measure is the minimal marrtingale measure $(\widehat{\mathbb{P}}=\widetilde{\mathbb{P}})$, then the operators $\widehat{\mathcal{A}}, \widehat{\mathcal{P}}, \widehat{x}, \widehat{y}, \widehat{\mathcal{G}}$ defined in Section 4.2 become identical to the operators $\widetilde{\mathcal{A}}, \widetilde{\mathcal{P}}, \widetilde{X}, \widetilde{y}, \widetilde{\mathcal{G}}$, respectively, as defined in Section 4.1. With $\widetilde{\mathbb{P}}$ as the chosen pricing measure, the first and second order correction terms in the approximation of $\Lambda$ simplify to:

$$
\begin{aligned}
\Lambda_{1}= & \frac{1}{2 \Lambda_{0}(\mathrm{~T}-t)} \int_{t}^{\mathrm{T}} \mathrm{d} t_{1}\left(\frac{1}{2} \lambda^{2}\right)_{1}\left(\widetilde{X}\left(t, t_{1}\right), \tilde{y}\left(t, t_{1}\right)\right), \\
\Lambda_{2}= & \frac{1}{2 \Lambda_{0}(\mathrm{~T}-t)}\left(\int_{t}^{\mathrm{T}} \mathrm{d} t_{1}\left(\frac{1}{2} \lambda^{2}\right)_{2}\left(\widetilde{X}\left(t, t_{1}\right), \tilde{y}\left(t, t_{1}\right)\right)+\int_{t}^{\mathrm{T}} \mathrm{d} t_{1} \widetilde{\mathcal{G}}_{1}\left(t, t_{1}\right) \int_{t_{1}}^{\mathrm{T}} \mathrm{d} t_{2}\left(\frac{1}{2} \lambda^{2}\right)_{1}\left(\widetilde{X}\left(t, t_{2}\right), \tilde{y}\left(t, t_{2}\right)\right)\right. \\
& -\left(1-\rho^{2}\right)\left(\frac{1}{2} \beta^{2}\right)_{0}\left[\left(\frac{1}{2} \lambda^{2}\right)_{0,1}^{2} \frac{1}{3}(\mathrm{~T}-t)^{3}+2 \gamma \nu \int_{t}^{\mathrm{T}} \mathrm{d} t_{1}\left(\frac{1}{2} \lambda^{2}\right)_{0,1}\left(\mathrm{~T}-t_{1}\right) \partial_{y}\left(\int_{t_{1}}^{\mathrm{T}} \mathrm{d} t_{2} \widetilde{\mathcal{G}}_{1}\left(t, t_{2}\right)\right) p^{\mathrm{BS}}(t)\right. \\
& +\gamma^{2} \nu^{2} \frac{\left(\frac{1}{2} \sigma^{2}\right)_{0,1}^{2}}{2 \pi \sigma_{0}^{2}} \int_{t}^{\mathrm{T}} \mathrm{d} t_{1} \frac{\left(\mathrm{T}-t_{1}\right)^{3 / 2}}{\sqrt{\mathrm{T}-t+t_{1}-t}} \exp \left(2 k-\frac{\left((k-x)+\frac{1}{2} \sigma_{0}^{2}(\mathrm{~T}-t)\right)^{2}}{\left.\left.\sigma_{0}^{2}\left(\mathrm{~T}-t+t_{1}-t\right)\right]-(\mathrm{T}-t) \frac{\Lambda_{1}^{2}}{2}\right),}\right.
\end{aligned}
$$

and the zeroth order term $\Lambda_{0}$ remains unaffected.

\section{$5 \quad$ Numerical Experiments}

In this section, we consider different local stochastic volatility models which provide different functional forms of $\lambda$. Our aim is to illustrate how the choice of instantaneous Sharpe ratio $\lambda$ affects the implied Sharpe ratio. Using our approximation result in Proposition 4, we discuss the practical implications. For the purpose of simplification of presentation, we will assume that the pricing measure $\widehat{\mathbb{P}}$ corresponds to the minimal martingale measure $\widetilde{\mathbb{P}}$.

\subsection{Heston model}

We first consider the famous Heston's stochastic volatility model, which under the physical measure $\mathbb{P}$, is given as

$$
\begin{aligned}
& \mathrm{dX}_{t}=\left(\lambda\left(\mathrm{X}_{t}, \mathrm{Y}_{t}\right) \sqrt{\mathrm{Y}_{t}}-\frac{1}{2} \mathrm{Y}_{t}\right) \mathrm{d} t+\sqrt{\mathrm{Y}_{t}} \mathrm{~dB}_{t}^{\mathrm{X}}, \\
& \mathrm{dY}_{t}=\left(\kappa\left(\theta-\mathrm{Y}_{t}\right)+\rho \delta \lambda\left(\mathrm{X}_{t}, \mathrm{Y}_{t}\right) \sqrt{\mathrm{Y}_{t}}\right) \mathrm{d} t+\delta \sqrt{\mathrm{Y}_{t}}\left(\rho \mathrm{dB}_{t}^{\mathrm{X}}+\sqrt{1-\rho_{t}^{2}} \mathrm{~dB}_{t}^{\mathrm{Y}}\right) .
\end{aligned}
$$

Comparing the above model with our formulation in (4), we have

$$
\begin{array}{llrl}
\mu(x, y) & =\lambda(x, y) \sqrt{y}, & & \sigma(y)=\sqrt{y} \\
c(x, y) & =\kappa(\theta-y)+\rho \delta \lambda(x, y) \sqrt{y}, & & \beta(y)=\delta \sqrt{y}
\end{array}
$$

We suppose the following form of $\lambda(x, y)=\frac{\sqrt{y}}{3}-\frac{\sqrt{\theta}}{2}$. This particular choice keeps the model in the affine class (see Duffee (2002)). Other forms of the function $\lambda(x, y)$ can also be considered as discussed in Section 5.3. For the chosen $\lambda$, we plot the second order approximation $\bar{\Lambda}_{2}$ of the implied Sharpe ratio in Figure 1 with respect to the risk-aversion parameter $\gamma$ for $\nu= \pm 1, \pm 2, \pm 3, \pm 4$. We observe that including European options in the investment portfolio increases the implied Sharpe ratio and its impact is greater for an investor with a higher value of $\gamma$. Thus, a risk-averse investor is better off including an European option in his portfolio when considering to invest using the utility maximisation approach. Due to the form of $\lambda$ which increases with the volatility of the underlying asset, an investor with high degree of risk-aversion $\gamma$ benefits more by including a European option in his investment portfolio compared to investing only in the underlying asset. 


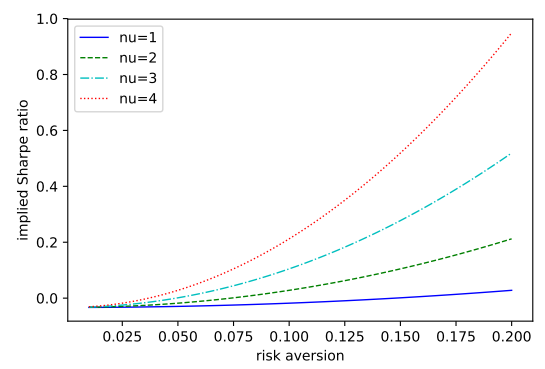

(a)

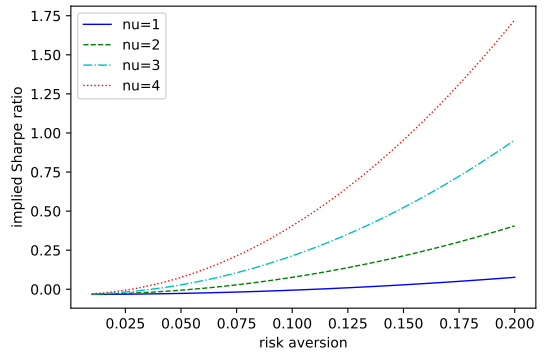

(b)

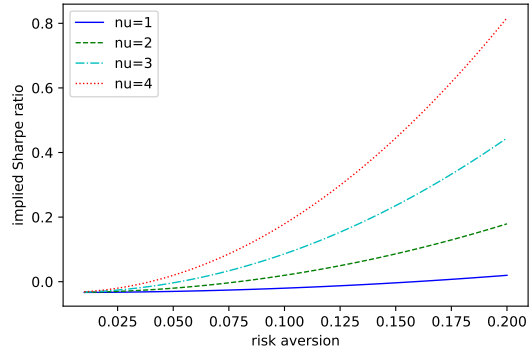

(c)

Figure 1: Implied Sharpe ratio for different values of $\log$ price (a) $x=\log (105)$ (b) $x=\log (100)$ (c) $x=\log (95)$. The parameter values used are $k=\log (100), t=0, \mathrm{~T}=4 / 52, \delta=0.2, \theta=0.04, \kappa=1.15, \rho=-0.4, \bar{x}=x, \bar{y}=\theta$, and $y=\bar{y}$.

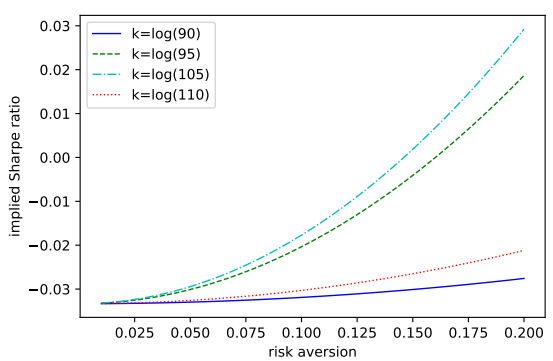

(a)

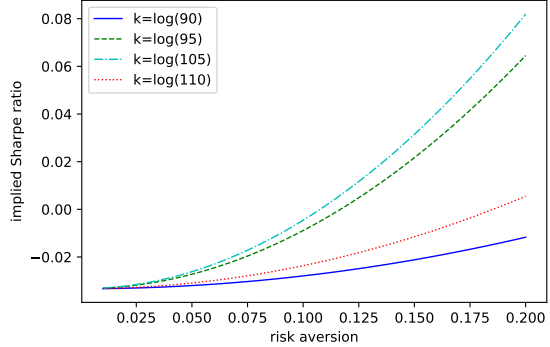

(b)

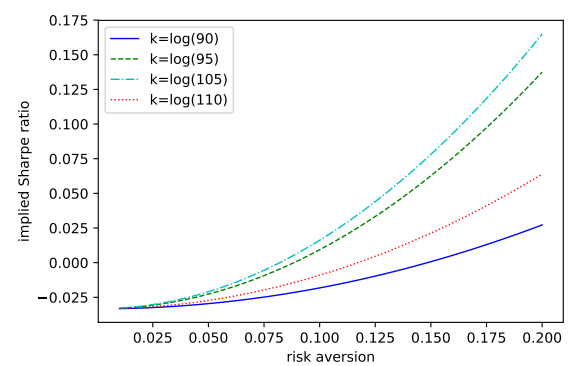

(c)

Figure 2: Relationship of the implied Sharpe ratio with respect to log-strike (a) $\mathrm{T}=4 / 52$ (b) $\mathrm{T}=6 / 52$ (c) $\mathrm{T}=9 / 52$. The parameter values used are $t=0, \nu=1, \delta=0.2, x=\log (100), \bar{x}=x, \theta=0.04, \kappa=1.15, \rho=-0.4, \bar{y}=\theta$, and $y=\bar{y}$.

To compare different European options we plot $\bar{\Lambda}_{2}$ with respect to the risk-aversion parameter $\gamma$ for different values of log-strike $k$ and maturity T in Figure 2 and Figure 3, respectively. In Figure 2, we observe that for a fixed maturity and the chosen parameter values, near-the-money European call options provide better implied Sharpe ratio than far-from-the-money European options. Moreover, this impact is more pronounced for an investor with higher value of $\gamma$. Thus, a risk-averse investor should consider to include near-the-money European options in the investment portfolio. The impact of option's moneyness on $\Lambda$ is through a non-linear term and thus, our second order approximation $\bar{\Lambda}_{2}$ plays a crucial role in establishing the role of option's moneyness on investor's investment preference. In Figure 3, we observe that for the chosen parameter values, the investor's implied Sharpe ratio increases with increasing maturity irrespective of the moneyness of the European call option, with an increasing impact for higher values of $\gamma$. Thus, under the Heston model with the chosen form of $\lambda$, the risk-averse investor should consider to include European options with longer time-to-maturity over options with shorter time-tomaturity. As the investor's investment horizon corresponds to the option's maturity, an investor will prefer long maturity options over short maturity to take advantage of stochastic volatility.

\subsection{Reciprocal Heston model}

We consider another stochastic volatility model, which under the physical measure $\mathbb{P}$ is given as

$$
\begin{aligned}
& \mathrm{dX}_{t}=\left(\mu-\frac{1}{2} \mathrm{Y}_{t}\right) \mathrm{d} t+\sqrt{\mathrm{Y}_{t}} \mathrm{~dB}_{t}^{\mathrm{X}} \\
& \mathrm{dY}_{t}=\left(a \mathrm{Y}_{t}+\frac{2\left(b^{2}-a \kappa\right)}{\mu^{2}(1-\rho)^{2}} \mathrm{Y}_{t}^{2}\right) \mathrm{d} t-\left(\frac{2}{1-\rho^{2}}\right)^{1 / 2} \frac{b}{\mu} \mathrm{Y}_{t}^{3 / 2}\left(\rho \mathrm{dB}_{t}^{\mathrm{X}}+\sqrt{1-\rho_{t}^{2}} \mathrm{~dB}_{t}^{\mathrm{Y}}\right)
\end{aligned}
$$




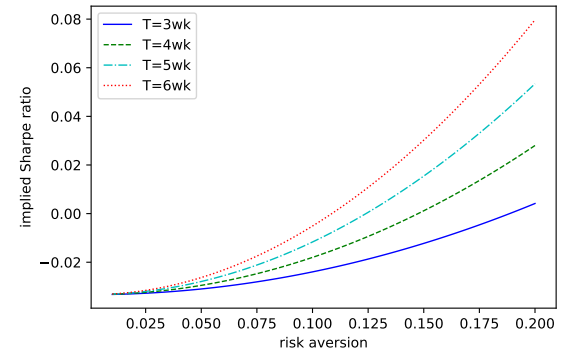

(a)

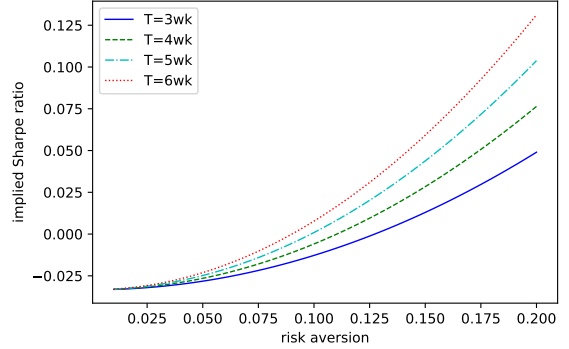

(b)

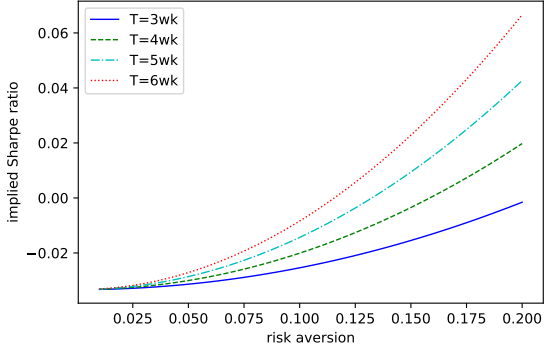

(c)

Figure 3: Relationship of the implied Sharpe ratio with respect to maturity (a) $x=\log (105)(\mathrm{b}) x=\log (100)(\mathrm{c})$ $x=\log (95)$. The parameter values used are $k=\log (100), t=0, \delta=0.2, \nu=1, \theta=0.04, \kappa=1.15, \rho=-0.4, \bar{x}=x, \bar{y}=\theta$, and $y=\bar{y}$.

The above model is referred as reciprocal Heston model as $\mathrm{Y}$ is the reciprocal of a Cox-Ingersoll-Rubinstein (CIR) process used in the Heston model. Comparing the above model with our formulation in (4), we get that

$$
\begin{array}{ll}
\mu(y)=\mu, & \sigma(y)=\sqrt{y}, \\
c(y)=a y+\frac{2\left(b^{2}-a \kappa\right)}{\mu^{2}(1-\rho)^{2}} y^{2}, & \beta(y)=-\left(\frac{2}{1-\rho^{2}}\right)^{1 / 2} \frac{b}{\mu} y^{3 / 2},
\end{array}
$$

where $(a, b, \kappa)$ must satisfy the usual Feller condition: $2 a \kappa \geq b^{2}$. This model leads to the functional choice of $\lambda(x, y)=\frac{\mu}{\sqrt{y}}$ which is different from the choice of $\lambda$ in Section 5.1. Once again, we plot $\bar{\Lambda}_{2}$ with respect to the risk-aversion parameter $\gamma$ in Figure 4. We observe that in the reciprocal Heston model, the implied Sharpe ratio of a risk-averse investor decreases by including the European option in the investment portfolio. This could be attributed to the form of $\lambda$ in the model which discourages an investor to include a European option in his investment portfolio.

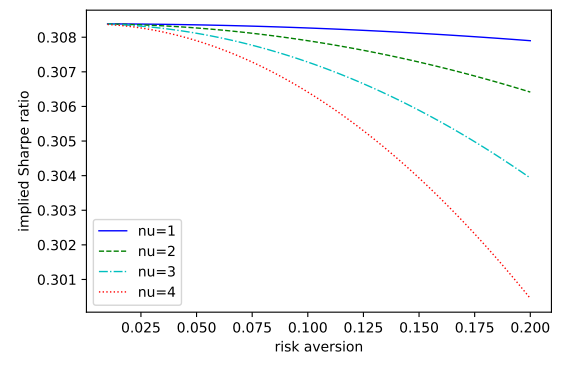

(a)

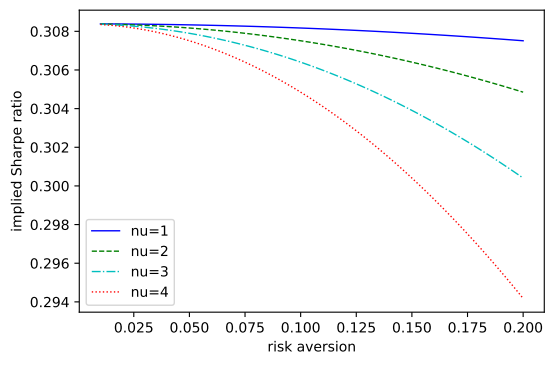

(b)

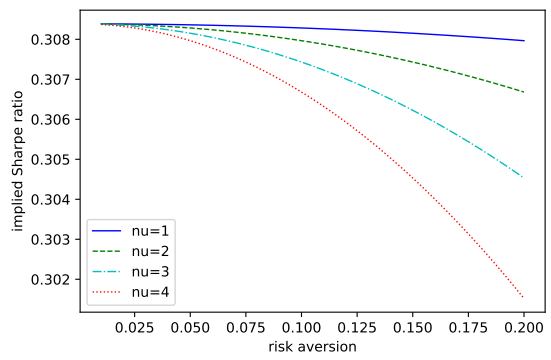

(c)

Figure 4: Implied Sharpe ratio for different values of $\log$ price (a) $x=\log (105)$ (b) $x=\log (100)$ (c) $x=\log (95)$. The parameter values used are $k=\log (100), t=0, \mathrm{~T}=4 / 52, \mu=0.05, a=5.0, b=0.04, \kappa=0.01, \rho=0.2, \bar{y}=0.04$, and $y=\bar{y}$. 


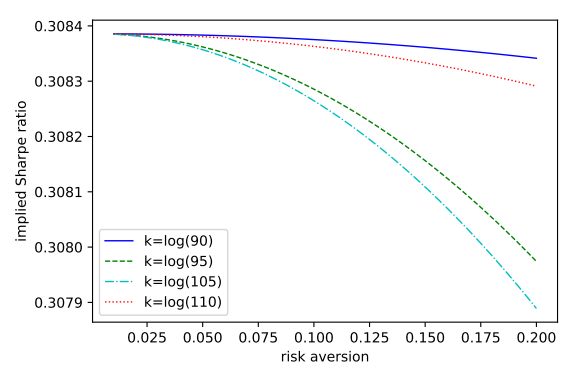

(a)

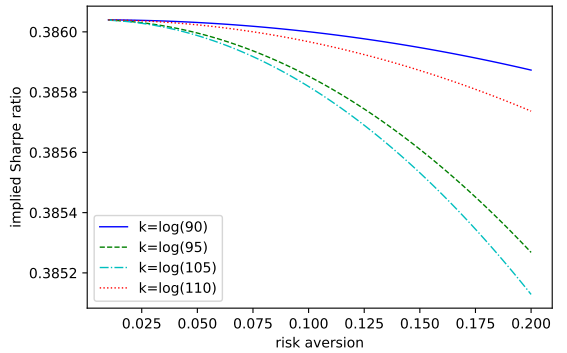

(b)

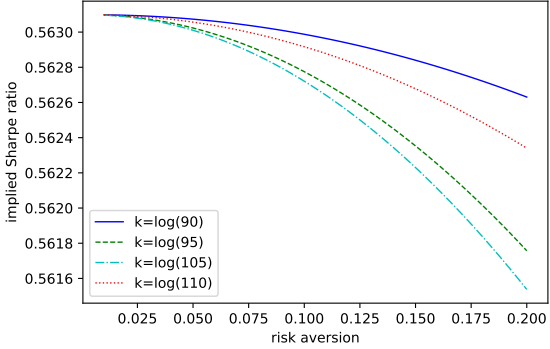

(c)

Figure 5: Relationship of the implied Sharpe ratio with respect to log-strike (a) $\mathrm{T}=4 / 52$ (b) $\mathrm{T}=6 / 52$ (c) $\mathrm{T}=9 / 52$. The parameter values used are $t=0, \nu=1, x=\log (100), \bar{x}=x, \mu=0.05, a=5.0, b=0.04, \kappa=0.01, \rho=0.2, \bar{y}=0.04$, and $y=\bar{y}$.

We also compare different European options by plotting $\bar{\Lambda}_{2}$ with respect to the risk-aversion parameter $\gamma$ for different values of log-strike $k$ and maturity $\mathrm{T}$ in Figure 5 and Figure 6, respectively. In Figure 5, we observe that for a fixed maturity and the chosen parameter values, far-from-the-money European call options reduce the implied Sharpe ratio less than near-the-money options. This finding is in agreement with our observation in Section 5.1 and reinforces the need to use our approximation $\bar{\Lambda}_{2}$ for comparing different European options for investment. In

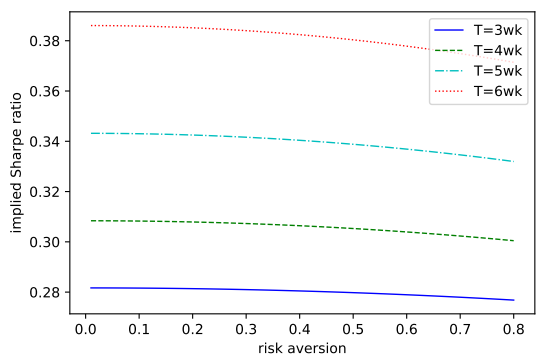

(a)

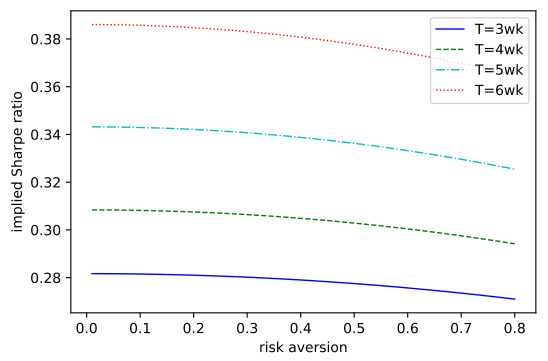

(b)

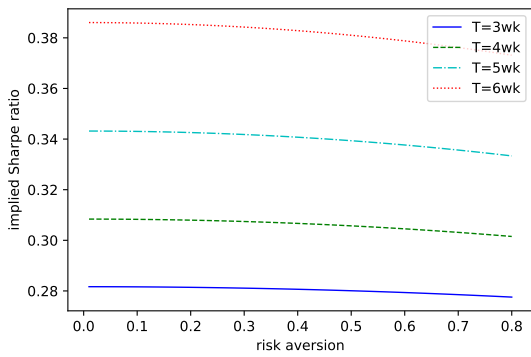

(c)

Figure 6: Relationship of the implied Sharpe ratio with respect to maturity (a) $x=\log (105)(\mathrm{b}) x=\log (100)(\mathrm{c}) x=\log (95)$. The parameter values used are $k=\log (100), t=0, \nu=1, \bar{x}=x, \mu=0.05, a=5.0, b=0.04, \kappa=0.01, \rho=0.2, \bar{y}=0.04$, and $y=\bar{y}$.

Figure 6, we once again observe that an investor prefers to use long maturity options in the investment portfolio. In the reciprocal Heston model, it is important to note that the risk-aversion parameter has little effect on the relative preference between different maturity options.

\subsection{Role of $\lambda$}

From our numerical studies in Section 5.1 abd 5.2, it is clear that the choice of instantaneous Sharpe ratio $\lambda$ plays a crucial role in the behaviour of the implied Sharpe ratio. In particular, we observe that in the Heston model, when $\lambda$ increases with volatility factor $Y$, an investor would prefer to add a European option to his investment portfolio. However, in the case of reciprocal Heston model where $\lambda$ decreases with volatility factor $Y$, an investor would prefer to invest only in the underlying asset. To test this observation further, we use $\lambda=\frac{0.05}{\sqrt{y}}$ in the Heston model considered in Section 5.1. With this particular choice, we plot $\bar{\Lambda}_{2}$ with respect to $\gamma$ in Figure 7 . We once again observe, that for the chosen $\lambda$ in the Heston model, an investor would prefer to invest only in the underlying risky asset. 


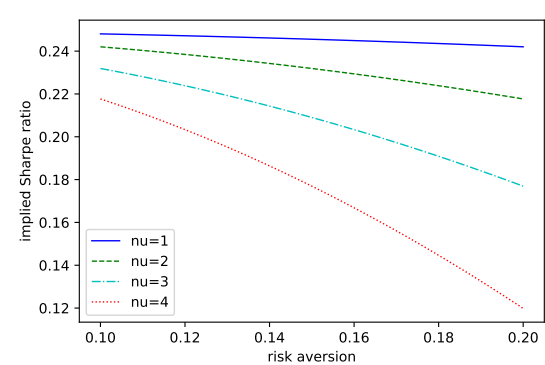

(a)

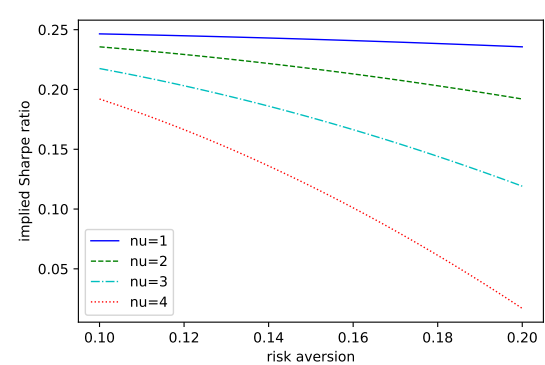

(b)

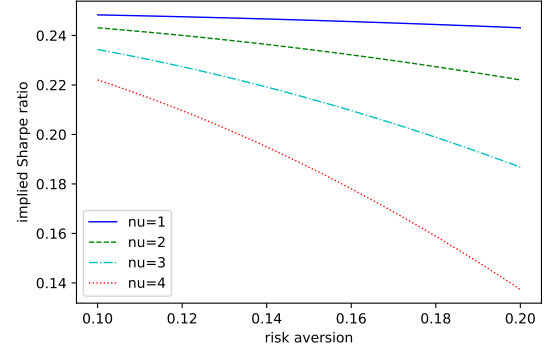

(c)

Figure 7: Implied Sharpe ratio for different values of $\log$ price (a) $x=\log (105)$ (b) $x=\log (100)$ (c) $x=\log (95)$. The parameter values used are $k=\log (100), t=0, \mathrm{~T}=4 / 52, \delta=0.2, \theta=0.04, \kappa=1.15, \rho=-0.4, \bar{x}=x, \bar{y}=\theta$, and $y=\bar{y}$.

\subsection{Accuracy of second-order approximations}

Using the results in Proposition 2 and 3, we demonstrate the accuracy of the asymptotic second-order approximations of $\psi$ and $p$ by comparing them with numerical solutions of PDEs (8) and (24), respectively.

In the case of Heston model, the true option price can also be obtained using the well-known formula based on the inverse Fourier transform (see, for example, Equation (10) Heston (1993)). We denote the option price thus obtained as "true" in Figure 8 and use it as a benchmark for our approximation and numerical solution of the PDE. We replace the operator $\widehat{\mathcal{A}}$ in $(24)$ with $\widetilde{\mathcal{A}}$ and implement an explicit finite-difference scheme to obtain the numerical solution. We keep the model parameters as used in Section 5.1. For the finite difference scheme, we use $\Delta t=\frac{\mathrm{T}}{4000}$ to discretise the time horizon, and use $\mathrm{I}=80$ steps and $\mathrm{J}=40$ steps to discretise the $x$-space and $y$-space, respectively.

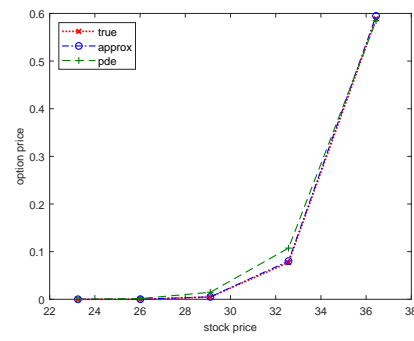

(a)

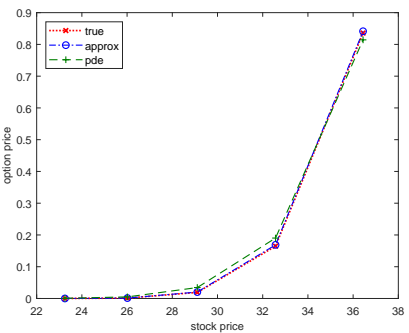

(d)

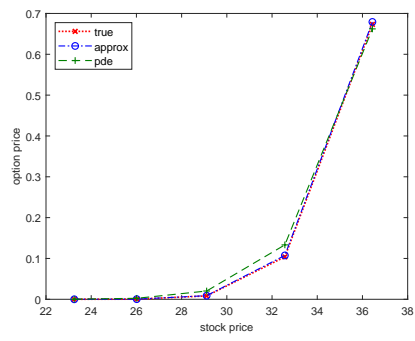

(b)

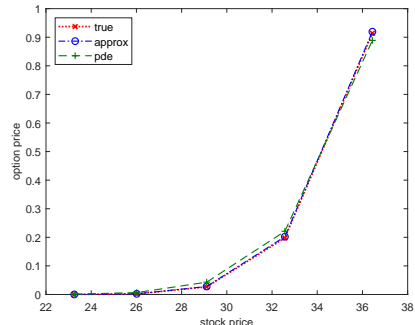

(e)

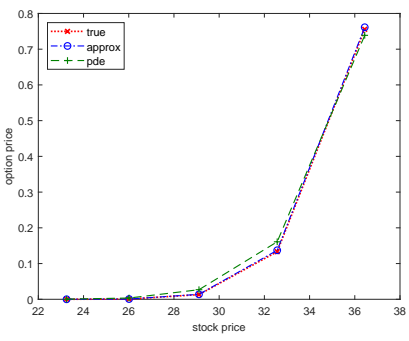

(c)

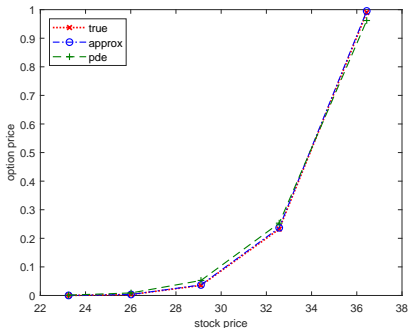

(f)

Figure 8: Option prices using - numerical integration (true), second order approximation formula (approx) and finite difference method (pde) - in the Heston model for $y=[0.2,0.225,0.25,0.275,0.3,0.325]$. Other parameter values used are $k=\log (40), t=0, \mathrm{~T}=4 / 52, \delta=0.2, \theta=0.04, \kappa=1.15, \rho=-0.4, \bar{x}=x$, and $\bar{y}=y$. 


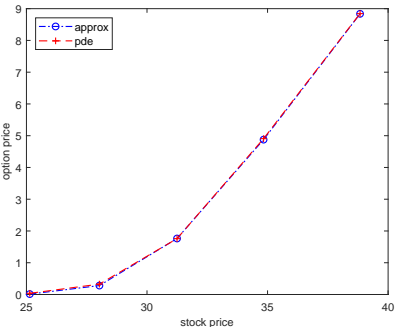

(a)

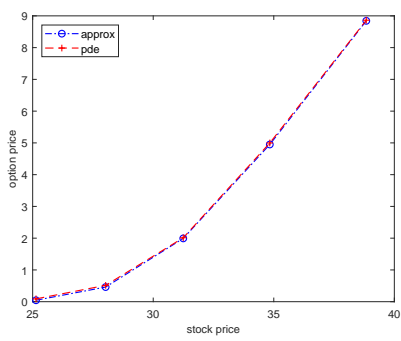

(d)

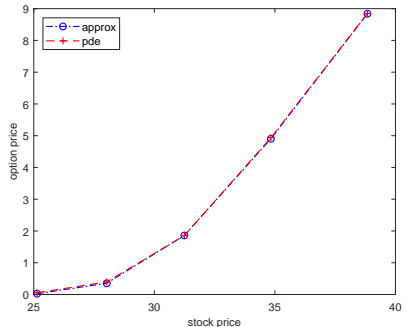

(b)

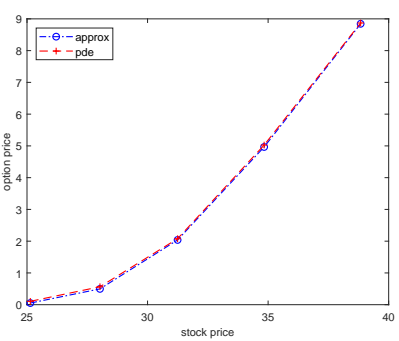

(e)

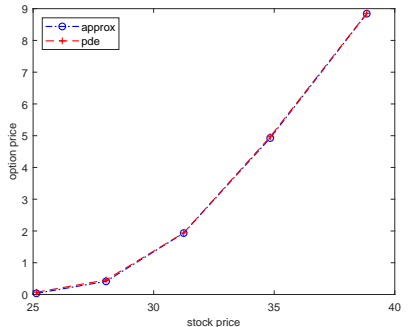

(c)

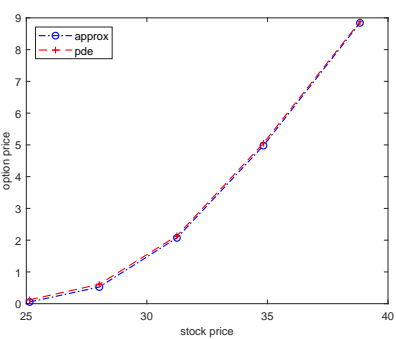

(f)

Figure 9: Option prices using - second order approximation formula (approx) and finite difference method (pde) - in the reciprocal Heston model for $y=[0.0918,0.1190,0.1463,0.1735,0.2008,0.2280]$. Other parameter values used are $k=$ $\log (30), t=0, \mathrm{~T}=4 / 52, \kappa=0.01, a=5, b=0.06, \mu=0.05, \rho=0.2, \bar{x}=x$, and $\bar{y}=y$.

We observe in Figure 8 that our second order approximation for option price, based on the formulas in Section $\mathrm{C}$, is in close agreement with the benchmark values. The numerical solutions from the finite-difference scheme are also close to the benchmark values, and can be improved upon by choosing more discretisation steps in the scheme. We also demonstrate the accuracy of our formula in the case of reciprocal Heston model. In the absence of a benchmark price formula, we only present the results obtained from the finite-difference scheme and second order approximation formulas in Figure 9. It is clear that for different values of the parameters, the two approximations remain in agreement.

Next, we compare the second order approximation of $\psi$ derived in Proposition 2 with the numerical solution obtained by solving the nonlinear PDE (8) for both Heston and reciprocal Heston model. In the absence of a closed-form solution for $\psi$, we only present the results obtained from the finite-difference scheme and second order approximation formulas for the Heston model in Figure 10 and for the reciprocal Heston model in Figure 11. It is clear that our approximation formula closely agrees with the numerical solution obtained by solving the PDE. 


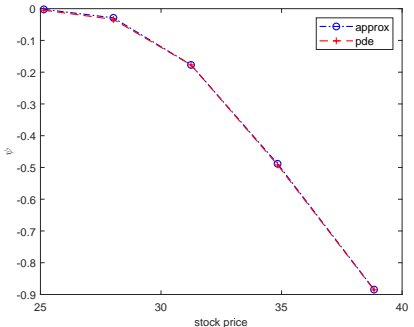

(a)

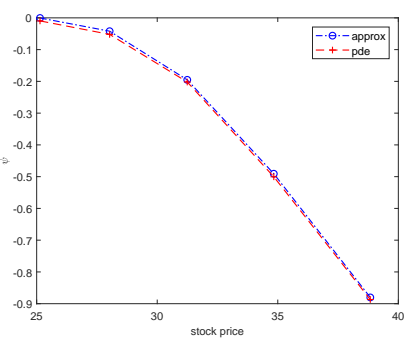

(d)

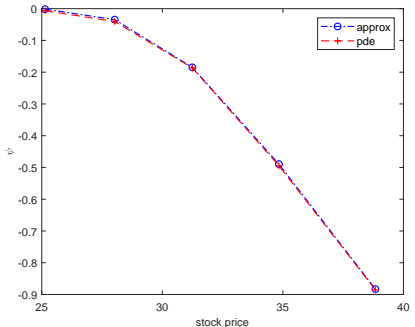

(b)

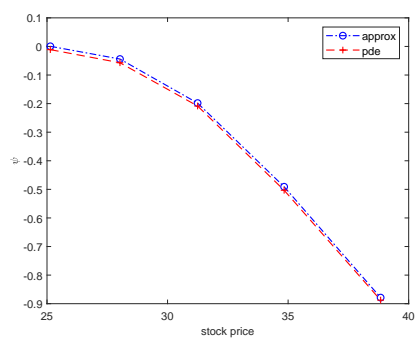

(e)

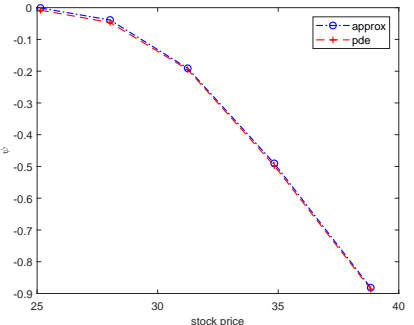

(c)

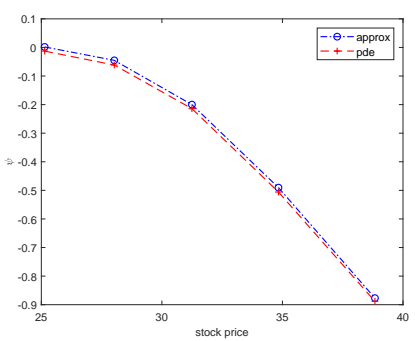

(f)

Figure 11: Plots for $\psi$ using - second order approximation formula (approx) and finite difference method (pde) - in the reciprocal Heston model for $y=[0.15,0.175,0.2,0.225,0.25,0.275]$. Other parameter values used are $\gamma=0.1, \nu=1, k=$ $\log (10), t=0, \mathrm{~T}=4 / 52, \kappa=0.01, a=5, b=0.06, \mu=0.05, \rho=0.2, \bar{x}=x$, and $\bar{y}=y$.

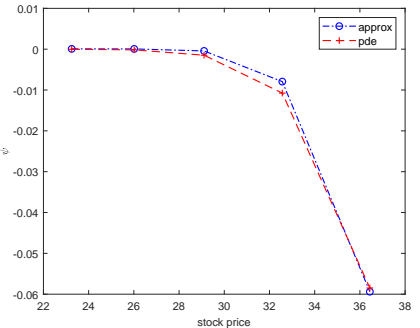

(a)

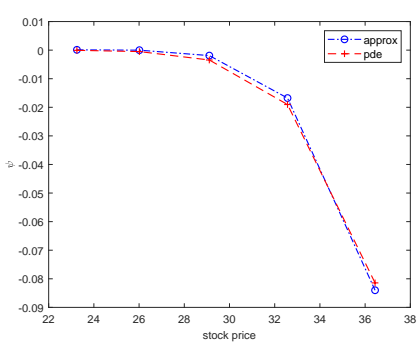

(d)

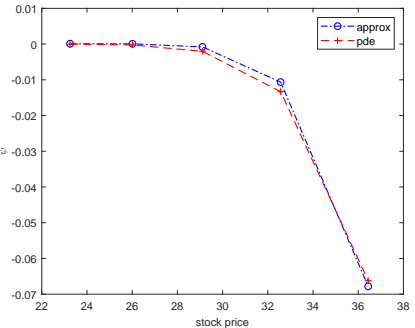

(b)

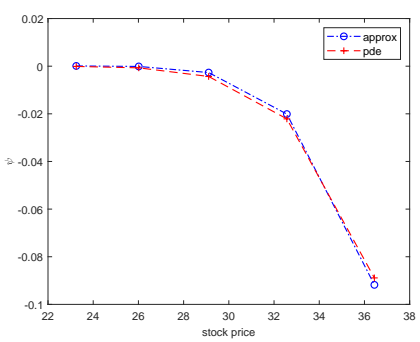

(e)

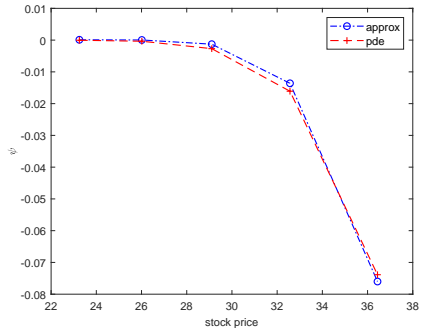

(c)

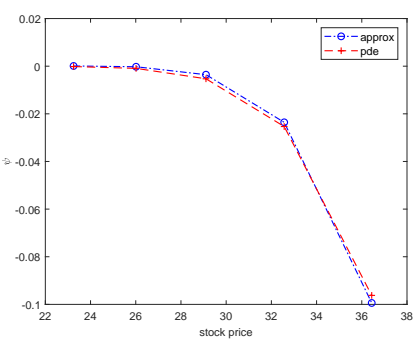

(f)

Figure 10: Plots for $\psi$ using - second order approximation formula (approx) and finite difference method (pde) - in the Heston model for $y=[0.2,0.225,0.25,0.275,0.3,0.325]$. Other parameter values used are $\gamma=0.1, \nu=1, k=\log (40), t=$ $0, \mathrm{~T}=4 / 52, \delta=0.2, \theta=0.04, \kappa=1.15, \rho=-0.4, \bar{x}=x$, and $\bar{y}=y$. 


\section{Conclusion}

In this work, we introduced a new concept of the implied Sharpe ratio which allows a risk-averse investor to compare the worthiness of different European options for investment. In a general market setting, we prove the existence and uniqueness of the implied Sharpe ratio and derive its asymptotic approximation formulas under general local stochastic volatility models. In two stochastic volatility model settings, we used the implied Sharpe ratio approximation formulas to observe that including a European option in the investment portfolio increases the investor's utility when the instantaneous Sharpe ratio increases with the volatility driving factor. Moreover, we observed that near-the-money and longer maturity options provide higher implied Sharpe ratio than far-fromthe-money and shorter maturity options, respectively.

\section{References}

Carmona, R. (2008). Indifference pricing: theory and applications. Princeton University Press.

Delbaen, F. and W. Schachermayer (2006). The mathematics of arbitrage. Springer Science \& Business Media.

Duffee, G. R. (2002). Term premia and interest rate forecasts in affine models. The Journal of Finance 57(1), 405-443.

Eeckhoudt, L., C. Gollier, and H. Schlesinger (1995). The risk-averse (and prudent) newsboy. Management science 41(5), 786-794.

Follmer, H. and M. Schweizer (1991). Hedging of contingent claims. Applied stochastic analysis 5, 389.

Friedman, A. (2008). Partial differential equations of parabolic type. Courier Dover Publications.

Heston, S. L. (1993). A closed-form solution for options with stochastic volatility with applications to bond and currency options. The review of financial studies $\underline{6}$ (2), 327-343.

Hodges, S. D. and A. Neuberger (1989). Optimal replication of contingent claims under transaction costs. The Review of Futures Markets 8(2), 222-239.

Jensen, M. C. (1969). Risk, the pricing of capital assets, and the evaluation of investment portfolios. Journal of business $\underline{42}(2), 167-247$.

Jewitt, I. (1987). Risk aversion and the choice between risky prospects: the preservation of comparative statics results. The Review of Economic Studies 54(1), 73-85.

Lorig, M. (2018). Indifference prices and implied volatilities. Mathematical Finance 28(1), 372-408.

Lorig, M., S. Pagliarani, and A. Pascucci (2015). Analytical expansions for parabolic equations. SIAM Journal on Applied Mathematics 75(2), 468-491.

Lorig, M., S. Pagliarani, and A. Pascucci (2017). Explicit implied volatilities for multifactor local-stochastic volatility models. Mathematical Finance 27(3), 926-960.

Merton, R. C. (1969). Lifetime portfolio selection under uncertainty: The continuous-time case. The Review of Economics and Statistics, 247-257.

Pagliarani, S. and A. Pascucci (2012). Analytical approximation of the transition density in a local volatility model. Central European Journal of Mathematics 10(1), 250-270.

Pham, H. (2009). Continuous-time stochastic control and optimization with financial applications, Volume 61. Springer Science \& Business Media.

Sharpe, W. F. (1966). Mutual fund performance. The Journal of Business 39(1), 119-138. 


\section{A Operator properties}

- The constant coefficient differential operator $\widetilde{\mathcal{A}}_{0}$

$$
\widetilde{\mathcal{A}}_{0}:=\left(\frac{1}{2} \sigma^{2}\right)_{0}\left(\partial_{x}^{2}-\partial_{x}\right)+(c-\rho \beta \lambda)_{0} \partial_{y}+\left(\frac{1}{2} \beta^{2}\right)_{0} \partial_{y}^{2}+(\rho \sigma \beta)_{0} \partial_{x} \partial_{y}
$$

gives rise to the semigroup $\widetilde{\mathcal{P}}_{0}\left(t, t_{1}\right)$ defined in (19). The semigroup $\widetilde{\mathcal{P}}_{0}\left(t, t_{1}\right)$ satisfies the following property:

$$
\widetilde{\mathcal{P}}_{0}\left(t, t_{1}\right) \widetilde{\mathcal{P}}_{0}\left(t_{1}, t_{2}\right)=\widetilde{\mathcal{P}}_{0}\left(t, t_{2}\right), \quad t \leq t_{1} \leq t_{2} .
$$

Moreover, by Duhamel's principle, the unique classical solution (if it exists) to any PDE of the form:

$$
0=\left(\partial_{t}+\widetilde{\mathcal{A}}_{0}\right) u+h, \quad u(\mathrm{~T}, x, y)=g(x, y),
$$

is given as

$$
u(t)=\widetilde{\mathcal{P}}_{0}(t, \mathrm{~T}) g+\int_{t}^{\mathrm{T}} \mathrm{d} t_{1} \widetilde{\mathcal{P}}_{0}\left(t, t_{1}\right) h\left(t_{1}\right)
$$

- For the operators $\widetilde{x}\left(t, t_{1}\right)$ and $\widetilde{y}\left(t, t_{1}\right)$ defined in (20) and (21), respectively, we can check by direct computations that they commute and have the following property

$$
\left(\widetilde{X}\left(t, t_{1}\right)\right)^{n}\left(\widetilde{y}\left(t, t_{1}\right)\right)^{m} \widetilde{\Gamma}_{0}\left(t, x, y ; t_{1}, x_{1}, y_{1}\right)=x_{1}^{n} y_{1}^{m} \widetilde{\Gamma}_{0}\left(t, x, y ; t_{1}, x_{1}, y_{1}\right), \quad n, m \in\{0\} \cup \mathbb{N} .
$$

Therefore, if function $f$ is a polynomial of $x$ and $y$, we have

$$
\begin{aligned}
\widetilde{\mathcal{P}}_{0}\left(t, t_{1}\right) f(x, y) & =\int_{\mathbb{R}^{2}} \mathrm{~d} x_{1} \mathrm{~d} y_{1} f\left(x_{1}, y_{1}\right) \widetilde{\Gamma}_{0}\left(t, x, y ; t_{1}, x_{1}, y_{1}\right) \\
& =f\left(\widetilde{x}\left(t, t_{1}\right), \widetilde{y}\left(t, t_{1}\right)\right) \int_{\mathbb{R}^{2}} \mathrm{~d} x_{1} \mathrm{~d} y_{1} \widetilde{\Gamma}_{0}\left(t, x, y ; t_{1}, x_{1}, y_{1}\right) \\
& =f\left(\widetilde{x}\left(t, t_{1}\right), \widetilde{y}\left(t, t_{1}\right)\right) .
\end{aligned}
$$

The following result is also satisfied by the operators defined in Section 4.1:

Lemma 5 (Lemma 3.7 Lorig (2018)). Let the operators $\widetilde{\mathcal{A}}_{n}, \widetilde{\mathcal{P}}_{0}\left(t, t_{1}\right), \widetilde{x}\left(t, t_{1}\right), \widetilde{y}\left(t, t_{1}\right)$ and $\widetilde{\mathcal{G}}_{n}\left(t, t_{1}\right)$ be given by (14), (19), (20), (21) and (22), respectively. Then, we have the following commutation-like relation

$$
\widetilde{\mathcal{P}}_{0}\left(t, t_{1}\right) \widetilde{\mathcal{A}}_{n}=\widetilde{\mathcal{G}}_{n}\left(t, t_{1}\right) \widetilde{\mathcal{P}}_{0}\left(t, t_{1}\right),
$$

where the operators act on measurable functions $f \in \mathrm{C}^{n+2}\left(\mathbb{R}^{2}\right)$ whose partial derivatives of all orders less than or equal to $n+2$ are measurable and at most exponentially growing.

\section{B Proofs}

In the following we have suppressed the dependence on $(x, y)$ and $\nu$ for notational convenience.

Proof of Proposition 2. By applying Duhamel's principle in (15), we see that the zeroth order term $\psi_{0}$ is given as

$$
\psi_{0}(t)=-\left(\frac{1}{2} \lambda^{2}\right)_{0}(\mathrm{~T}-t)-\gamma \nu p^{\mathrm{BS}}(t)
$$

where

$$
p^{\mathrm{BS}}(t):=\mathrm{e}^{x} \Phi\left(\frac{1}{\sigma_{0} \sqrt{\mathrm{T}-t}}\left(x-k+\frac{1}{2} \sigma_{0}^{2}(\mathrm{~T}-t)\right)\right)-\mathrm{e}^{k} \Phi\left(\frac{1}{\sigma_{0} \sqrt{\mathrm{T}-t}}\left(x-k-\frac{1}{2} \sigma_{0}^{2}(\mathrm{~T}-t)\right)\right) .
$$


$p^{\mathrm{BS}}$ denotes the price of a European call option with strike $\exp (k)$ and time to maturity $\mathrm{T}-t$, in a Black-Scholes model with starting asset price $\exp (x)$ and volatility coefficient $\sigma_{0}$. For the first order term, we have

$$
0=\left(\partial_{t}+\widetilde{\mathcal{A}}_{0}\right) \psi_{1}+\widetilde{\mathcal{A}}_{1} \psi_{0}-\left(\frac{1}{2} \lambda^{2}\right)_{1}, \quad \psi_{1}(\mathrm{~T})=0
$$

Again by applying Duhamel's principle, the results in Lemma 5 and (35), we get

$$
\begin{aligned}
\psi_{1}(t) & =\int_{t}^{\mathrm{T}} \mathrm{d} t_{1} \widetilde{\mathcal{P}}_{0}\left(t, t_{1}\right)\left(\widetilde{\mathcal{A}}_{1} \psi_{0}\left(t_{1}\right)-\left(\frac{1}{2} \lambda^{2}\right)_{1}\right) \\
& =\int_{t}^{\mathrm{T}} \mathrm{d} t_{1} \widetilde{\mathcal{P}}_{0}\left(t, t_{1}\right)\left(\widetilde{\mathcal{A}}_{1}\left(-\left(\frac{1}{2} \lambda^{2}\right)_{0}\left(\mathrm{~T}-t_{1}\right)-\gamma \nu p^{\mathrm{BS}}\left(t_{1}\right)\right)-\left(\frac{1}{2} \lambda^{2}\right)_{1}\right) \\
& =-\int_{t}^{\mathrm{T}} \mathrm{d} t_{1} \widetilde{\mathcal{P}}_{0}\left(t, t_{1}\right) \widetilde{\mathcal{A}}_{1} \gamma \nu p^{\mathrm{BS}}\left(t_{1}\right)-\int_{t}^{\mathrm{T}} \mathrm{d} t_{1}\left(\frac{1}{2} \lambda^{2}\right)_{1}\left(\widetilde{x}\left(t, t_{1}\right), \widetilde{y}\left(t, t_{1}\right)\right) \\
& =-\gamma \nu \int_{t}^{\mathrm{T}} \mathrm{d} t_{1} \widetilde{\mathcal{G}}_{1}\left(t, t_{1}\right) \widetilde{\mathcal{P}}_{0}\left(t, t_{1}\right) p^{\mathrm{BS}}\left(t_{1}\right)-\int_{t}^{\mathrm{T}} \mathrm{d} t_{1}\left(\frac{1}{2} \lambda^{2}\right)_{1}\left(\widetilde{\mathcal{X}}\left(t, t_{1}\right), \widetilde{y}\left(t, t_{1}\right)\right) \\
& =-\gamma \nu \int_{t}^{\mathrm{T}} \mathrm{d} t_{1} \widetilde{\mathcal{G}}_{1}\left(t, t_{1}\right) p^{\mathrm{BS}}(t)-\int_{t}^{\mathrm{T}} \mathrm{d} t_{1}\left(\frac{1}{2} \lambda^{2}\right)_{1}\left(\widetilde{\mathcal{X}}\left(t, t_{1}\right), \widetilde{y}\left(t, t_{1}\right)\right) .
\end{aligned}
$$

In the above, we have also used the semi-group property (33) of $\widetilde{\mathcal{P}}_{0}$. For the second order term, we have

$$
0=\left(\partial_{t}+\widetilde{\mathcal{A}}_{0}\right) \psi_{2}+\widetilde{\mathcal{A}}_{2} \psi_{0}+\widetilde{\mathcal{A}}_{1} \psi_{1}-\left(\frac{1}{2} \lambda^{2}\right)_{2}+\left(1-\rho^{2}\right)\left(\frac{1}{2} \beta^{2}\right)_{0}\left(\partial_{y} \psi_{1}\right)^{2}, \quad \psi_{2}(\mathrm{~T})=0
$$

Once more, by Duhamel's principle, we obtain

$$
\begin{aligned}
\psi_{2}(t) & =\int_{t}^{\mathrm{T}} \mathrm{d} t_{1} \widetilde{\mathcal{P}}_{0}\left(t, t_{1}\right) \widetilde{\mathcal{A}}_{2} \psi_{0}\left(t_{1}\right)+\int_{t}^{\mathrm{T}} \mathrm{d} t_{1} \widetilde{\mathcal{P}}_{0}\left(t, t_{1}\right) \widetilde{\mathcal{A}}_{1} \psi_{1}\left(t_{1}\right) \\
& -\int_{t}^{\mathrm{T}} \mathrm{d} t_{1} \widetilde{\mathcal{P}}_{0}\left(t, t_{1}\right)\left(\frac{1}{2} \lambda^{2}\right)_{2}+\int_{t}^{\mathrm{T}} \mathrm{d} t_{1} \widetilde{\mathcal{P}}_{0}\left(t, t_{1}\right)\left(1-\rho^{2}\right)\left(\frac{1}{2} \beta^{2}\right)_{0}\left(\partial_{y} \psi_{1}\left(t_{1}\right)\right)^{2} \\
& =: \mathrm{I}+\mathrm{II}+\mathrm{III}+\mathrm{IV} .
\end{aligned}
$$

In term I above, we note the following while using the results in Lemma 5 and the semi-group property (33) of $\widetilde{\mathcal{P}}_{0}$ :

$$
\begin{aligned}
\int_{t}^{\mathrm{T}} \mathrm{d} t_{1} \widetilde{\mathcal{P}}_{0}\left(t, t_{1}\right) \widetilde{\mathcal{A}}_{2} \psi_{0}\left(t_{1}\right) & =\int_{t}^{\mathrm{T}} \mathrm{d} t_{1} \widetilde{\mathcal{P}}_{0}\left(t, t_{1}\right) \widetilde{\mathcal{A}}_{2}\left(-\left(\frac{1}{2} \lambda^{2}\right)_{0}(\mathrm{~T}-t)-\gamma \nu p^{\mathrm{BS}}\left(t_{1}\right)\right) \\
& =-\gamma \nu \int_{t}^{\mathrm{T}} \mathrm{d} t_{1} \widetilde{\mathcal{P}}_{0}\left(t, t_{1}\right) \widetilde{\mathcal{A}}_{2} p^{\mathrm{BS}}\left(t_{1}\right) \\
& =-\gamma \nu \int_{t}^{\mathrm{T}} \mathrm{d} t_{1} \widetilde{\mathcal{G}}_{2}\left(t, t_{1}\right) \widetilde{\mathcal{P}}_{0}\left(t, t_{1}\right) \widetilde{\mathcal{P}}_{0}\left(t_{1}, \mathrm{~T}\right) \varphi \\
& =-\gamma \nu \int_{t}^{\mathrm{T}} \mathrm{d} t_{1} \widetilde{\mathcal{G}}_{2}\left(t, t_{1}\right) \widetilde{\mathcal{P}}_{0}(t, \mathrm{~T}) \varphi \\
& =-\gamma \nu \int_{t}^{\mathrm{T}} \mathrm{d} t_{1} \widetilde{\mathcal{G}}_{2}\left(t, t_{1}\right) p^{\mathrm{BS}}(t)
\end{aligned}
$$

Next, for term II in (37), we have the following from (36)

$$
\int_{t}^{\mathrm{T}} \mathrm{d} t_{1} \widetilde{\mathcal{P}}_{0}\left(t, t_{1}\right) \widetilde{\mathcal{A}}_{1} \psi_{1}\left(t_{1}\right)=\int_{t}^{\mathrm{T}} \mathrm{d} t_{1} \widetilde{\mathcal{P}}_{0}\left(t, t_{1}\right) \widetilde{\mathcal{A}}_{1}\left(-\gamma \nu \int_{t_{1}}^{\mathrm{T}} \mathrm{d} t_{2} \widetilde{\mathcal{G}}_{1}\left(t_{1}, t_{2}\right) p^{\mathrm{BS}}\left(t_{1}\right)-\int_{t_{1}}^{\mathrm{T}} \mathrm{d} t_{2}\left(\frac{1}{2} \lambda^{2}\right)_{1}\left(\widetilde{X}\left(t_{1}, t_{2}\right), \widetilde{y}\left(t_{1}, t_{2}\right)\right)\right) .
$$

The first term above can be computed by using Lemma 5 and property in (33)

$$
-\gamma \nu \int_{t}^{\mathrm{T}} \mathrm{d} t_{1} \widetilde{\mathcal{P}}_{0}\left(t, t_{1}\right) \widetilde{\mathcal{A}}_{1} \int_{t_{1}}^{\mathrm{T}} \mathrm{d} t_{2} \widetilde{\mathcal{G}}_{1}\left(t_{1}, t_{2}\right) p^{\mathrm{BS}}\left(t_{1}\right)=-\gamma \nu \int_{t}^{\mathrm{T}} \mathrm{d} t_{1} \widetilde{\mathcal{G}}_{1}\left(t, t_{1}\right) \widetilde{\mathcal{P}}_{0}\left(t, t_{1}\right) \int_{t_{1}}^{\mathrm{T}} \mathrm{d} t_{2} \widetilde{\mathcal{G}}_{1}\left(t_{1}, t_{2}\right) \widetilde{\mathcal{P}}_{0}\left(t_{1}, \mathrm{~T}\right) \varphi
$$




$$
\begin{aligned}
& =-\gamma \nu \int_{t}^{\mathrm{T}} \mathrm{d} t_{1} \widetilde{\mathcal{G}}_{1}\left(t, t_{1}\right) \int_{t_{1}}^{\mathrm{T}} \mathrm{d} t_{2} \widetilde{\mathcal{G}}_{1}\left(t_{1}, t_{2}\right) \widetilde{\mathcal{P}}_{0}\left(t, t_{1}\right) \widetilde{\mathcal{P}}_{0}\left(t_{1}, \mathrm{~T}\right) \varphi \\
& =-\gamma \nu \int_{t}^{\mathrm{T}} \mathrm{d} t_{1} \widetilde{\mathcal{G}}_{1}\left(t, t_{1}\right) \int_{t_{1}}^{\mathrm{T}} \mathrm{d} t_{2} \widetilde{\mathcal{G}}_{1}\left(t_{1}, t_{2}\right) p^{\mathrm{BS}}(t) .
\end{aligned}
$$

The second term in (38) can be similarly resolved as

$$
\begin{aligned}
-\int_{t}^{\mathrm{T}} \mathrm{d} t_{1} \widetilde{\mathcal{P}}_{0}\left(t, t_{1}\right) \widetilde{\mathcal{A}}_{1} \int_{t_{1}}^{\mathrm{T}} \mathrm{d} t_{2}\left(\frac{1}{2} \lambda^{2}\right)_{1}\left(\widetilde{X}\left(t_{1}, t_{2}\right), \widetilde{y}\left(t_{1}, t_{2}\right)\right) & =-\int_{t}^{\mathrm{T}} \mathrm{d} t_{1} \widetilde{\mathcal{G}}_{1}\left(t, t_{1}\right) \widetilde{\mathcal{P}}_{0}\left(t, t_{1}\right) \int_{t_{1}}^{\mathrm{T}} \mathrm{d} t_{2} \widetilde{\mathcal{P}}_{0}\left(t_{1}, t_{2}\right)\left(\frac{1}{2} \lambda^{2}\right)_{1} \\
& =-\int_{t}^{\mathrm{T}} \mathrm{d} t_{1} \widetilde{\mathcal{G}}_{1}\left(t, t_{1}\right) \int_{t_{1}}^{\mathrm{T}} \mathrm{d} t_{2} \widetilde{\mathcal{P}}_{0}\left(t, t_{2}\right)\left(\frac{1}{2} \lambda^{2}\right)_{1} \\
& =-\int_{t}^{\mathrm{T}} \mathrm{d} t_{1} \widetilde{\mathcal{G}}_{1}\left(t, t_{1}\right) \int_{t_{1}}^{\mathrm{T}} \mathrm{d} t_{2}\left(\frac{1}{2} \lambda^{2}\right)_{1}\left(\widetilde{X}\left(t, t_{2}\right), \widetilde{y}\left(t, t_{2}\right)\right) .
\end{aligned}
$$

Term III in (37) can be computed using the property in (35):

$$
-\int_{t}^{\mathrm{T}} \mathrm{d} t_{1} \widetilde{\mathcal{P}}_{0}\left(t, t_{1}\right)\left(\frac{1}{2} \lambda^{2}\right)_{2}=-\int_{t}^{\mathrm{T}} \mathrm{d} t_{1}\left(\frac{1}{2} \lambda^{2}\right)_{2}\left(\widetilde{X}\left(t, t_{1}\right), \widetilde{y}\left(t, t_{1}\right)\right) .
$$

Term IV in (37) is computed as

$$
\begin{aligned}
& \int_{t}^{\mathrm{T}} \mathrm{d} t_{1} \widetilde{\mathcal{P}}_{0}\left(t, t_{1}\right)\left(1-\rho^{2}\right)\left(\frac{1}{2} \beta^{2}\right)_{0}\left(\partial_{y} \psi_{1}\left(t_{1}\right)\right)^{2} \\
& =\left(1-\rho^{2}\right)\left(\frac{1}{2} \beta^{2}\right)_{0} \int_{t}^{\mathrm{T}} \mathrm{d} t_{1} \widetilde{\mathcal{P}}_{0}\left(t, t_{1}\right)\left[\gamma^{2} \nu^{2}\left(\partial_{y} \zeta_{1}\left(t_{1}\right)\right)^{2}+\left(\partial_{y} \eta\left(t_{1}\right)\right)^{2}-2 \gamma \nu \partial_{y} \zeta_{1}\left(t_{1}\right) \partial_{y} \eta\left(t_{1}\right)\right]
\end{aligned}
$$

where we have defined $\zeta(t)$ and $\eta(t)$ as

$$
\zeta(t):=\int_{t}^{\mathrm{T}} \mathrm{d} t_{1} \widetilde{\mathcal{G}}_{1}\left(t, t_{1}\right) p^{\mathrm{BS}}(t), \quad \eta(t):=-\int_{t}^{\mathrm{T}} \mathrm{d} t_{1}\left(\frac{1}{2} \lambda^{2}\right)_{1}\left(\widetilde{X}\left(t, t_{1}\right), \widetilde{y}\left(t, t_{1}\right)\right) .
$$

By straightforward computations, we have that

$$
\partial_{y} \eta\left(t_{1}\right)=-\int_{t_{1}}^{\mathrm{T}} \mathrm{d} t_{2}\left(\frac{1}{2} \lambda^{2}\right)_{0,1}=-\left(\frac{1}{2} \lambda^{2}\right)_{0,1}\left(\mathrm{~T}-t_{1}\right) .
$$

Next, we use the properties in (34) and (33) to obtain the following:

$$
\begin{aligned}
\int_{t}^{\mathrm{T}} \mathrm{d} t_{1} \widetilde{\mathcal{P}}_{0}\left(t, t_{1}\right) \partial_{y} \zeta_{1}\left(t_{1}\right) \partial_{y} \eta\left(t_{1}\right) & =-\int_{t}^{\mathrm{T}} \mathrm{d} t_{1}\left(\frac{1}{2} \lambda^{2}\right)_{0,1}\left(\mathrm{~T}-t_{1}\right) \widetilde{\mathcal{P}}_{0}\left(t, t_{1}\right) \partial_{y} \zeta\left(t_{1}\right) \\
& =-\int_{t}^{\mathrm{T}} \mathrm{d} t_{1}\left(\frac{1}{2} \lambda^{2}\right)_{0,1}\left(\mathrm{~T}-t_{1}\right) \partial_{y}\left(\widetilde{\mathcal{P}}_{0}\left(t, t_{1}\right) \int_{t_{1}}^{\mathrm{T}} \mathrm{d} t_{2} \widetilde{\mathcal{G}}_{1}\left(t_{1}, t_{2}\right) \widetilde{\mathcal{P}}_{0}\left(t_{1}, t_{2}\right) p^{\mathrm{BS}}\left(t_{2}\right)\right) \\
& =-\int_{t}^{\mathrm{T}} \mathrm{d} t_{1}\left(\frac{1}{2} \lambda^{2}\right)_{0,1}\left(\mathrm{~T}-t_{1}\right) \partial_{y}\left(\widetilde{\mathcal{P}}_{0}\left(t, t_{1}\right) \int_{t_{1}}^{\mathrm{T}} \mathrm{d} t_{2} \widetilde{\mathcal{P}}_{0}\left(t_{1}, t_{2}\right) \widetilde{\mathcal{A}}_{1} p^{\mathrm{BS}}\left(t_{2}\right)\right) \\
& =-\int_{t}^{\mathrm{T}} \mathrm{d} t_{1}\left(\frac{1}{2} \lambda^{2}\right)_{0,1}\left(\mathrm{~T}-t_{1}\right) \partial_{y}\left(\int_{t_{1}}^{\mathrm{T}} \mathrm{d} t_{2} \widetilde{\mathcal{G}}_{1}\left(t, t_{2}\right) p^{\mathrm{BS}}(t)\right) .
\end{aligned}
$$

To tackle the final term in (39), we note the following

$$
\partial_{y} \zeta\left(t_{1}\right)=\partial_{y}\left(\int_{t_{1}}^{\mathrm{T}} \mathrm{d} t_{2} \widetilde{\mathcal{G}}_{1}\left(t_{1}, t_{2}\right)\right) p^{\mathrm{BS}}\left(t_{1}\right)=\left(\mathrm{T}-t_{1}\right)\left(\frac{1}{2} \sigma^{2}\right)_{0,1}\left(\partial_{x}^{2}-\partial_{x}\right) p^{\mathrm{BS}}\left(t_{1}\right) .
$$

Using the explicit expression for $p^{\mathrm{BS}}(t)$, we obtain

$$
\left(\partial_{x}^{2}-\partial_{x}\right) p^{\mathrm{BS}}\left(t_{1}\right)=\frac{1}{\sigma_{0} \sqrt{\mathrm{T}-t_{1}}} \mathrm{e}^{x} \phi\left(d_{+}\left(t_{1}\right)\right),
$$


where $\phi$ is the density function of standard normal distribution. Then, from the calculations in Appendix B of Lorig (2018), we obtain the following

$$
\begin{aligned}
& \int_{t}^{\mathrm{T}} \mathrm{d} t_{1} \widetilde{\mathcal{P}}_{0}\left(t, t_{1}\right)\left(\partial_{y} \zeta\left(t_{1}\right)\right)^{2} \\
& =\frac{\left(\frac{1}{2} \sigma^{2}\right)_{0,1}^{2}}{2 \pi \sigma_{0}^{2}} \int_{t}^{\mathrm{T}} \mathrm{d} t_{1} \frac{\left(\mathrm{T}-t_{1}\right)^{3 / 2}}{\sqrt{\mathrm{T}-t+t_{1}-t}} \exp \left(2 k-\frac{\left((k-x)+\frac{1}{2} \sigma_{0}^{2}(\mathrm{~T}-t)\right)^{2}}{\sigma_{0}^{2}\left(\mathrm{~T}-t+t_{1}-t\right)}\right) .
\end{aligned}
$$

Proof of Proposition 3. The linear operator $\widehat{\mathcal{A}}_{0}$ is the infinitesimal generator of a diffusion in $\mathbb{R}^{2}$ whose drift vector and covariance matrix are constant. Then, by Duhamel's principle, the zeroth order term $p_{0}$, which is independent of $y$, is given as

$$
p_{0}(t)=p^{\mathrm{BS}}(t)
$$

where for standard normal cdf $\Phi$, we have

$$
p^{\mathrm{BS}}(t):=\mathrm{e}^{x} \Phi\left(\frac{1}{\sigma_{0} \sqrt{\mathrm{T}-t}}\left(x-k+\frac{1}{2} \sigma_{0}^{2}(\mathrm{~T}-t)\right)\right)-\mathrm{e}^{k} \Phi\left(\frac{1}{\sigma_{0} \sqrt{\mathrm{T}-t}}\left(x-k-\frac{1}{2} \sigma_{0}^{2}(\mathrm{~T}-t)\right)\right) .
$$

For the first order term, we have the following equation

$$
\left(\partial_{t}+\widehat{\mathcal{A}}_{0}\right) p_{1}+\widehat{\mathcal{A}}_{1} p_{0}=0, \quad p_{1}(\mathrm{~T})=0 .
$$

Once again, by using Duhamel's principle, we obtain that

$$
p_{1}(t)=\int_{t}^{\mathrm{T}} \mathrm{d} t_{1} \widehat{\mathcal{P}}_{0}\left(t, t_{1}\right) \widehat{\mathcal{A}}_{1} p_{0}\left(t_{1}\right)
$$

Then, by applying the result in Lemma 5, we get

$$
\begin{aligned}
p_{1}(t) & =\int_{t}^{\mathrm{T}} \mathrm{d} t_{1} \widehat{\mathcal{G}}_{1}\left(t, t_{1}\right) \widehat{\mathcal{P}}_{0}\left(t, t_{1}\right) p_{0}\left(t_{1}\right) \\
& =\int_{t}^{\mathrm{T}} \mathrm{d} t_{1} \widehat{\mathcal{G}}_{1}\left(t, t_{1}\right) \widehat{\mathcal{P}}_{0}\left(t, t_{1}\right) \widehat{\mathcal{P}}_{0}\left(t_{1}, \mathrm{~T}\right) \varphi \\
& =\int_{t}^{\mathrm{T}} \mathrm{d} t_{1} \widehat{\mathcal{G}}_{1}\left(t, t_{1}\right) p_{0}(t),
\end{aligned}
$$

Now, for the second order term, we have

$$
\left(\partial_{t}+\widehat{\mathcal{A}}_{0}\right) p_{2}+\widehat{\mathcal{A}}_{1} p_{1}+\widehat{\mathcal{A}}_{2} p_{0}=0, \quad p_{2}(\mathrm{~T})=0 .
$$

By applying Duhamel's principle, we get

$$
p_{2}(t)=\int_{t}^{\mathrm{T}} \mathrm{d} t_{1} \widehat{\mathcal{P}}_{0}\left(t, t_{1}\right)\left(\widehat{\mathcal{A}}_{1} p_{1}\left(t_{1}\right)+\widehat{\mathcal{A}}_{2} p_{0}\left(t_{1}\right)\right) .
$$

From the result in Lemma 5 and the result for $p_{1}$, we obtain that

$$
\begin{aligned}
p_{2}(t) & =\int_{t}^{\mathrm{T}} \mathrm{d} t_{1} \widehat{\mathcal{P}}_{0}\left(t, t_{1}\right) \widehat{\mathcal{A}}_{1} \int_{t_{1}}^{\mathrm{T}} \mathrm{d} t_{2} \widehat{\mathcal{P}}_{0}\left(t_{1}, t_{2}\right) \widehat{\mathcal{A}}_{1} p_{0}\left(t_{2}\right) \\
& +\int_{t}^{\mathrm{T}} \mathrm{d} t_{1} \widehat{\mathcal{S}}_{2}\left(t, t_{1}\right) \widehat{\mathcal{P}}_{0}\left(t, t_{1}\right) p_{0}\left(t_{1}\right)
\end{aligned}
$$




$$
\begin{aligned}
& =\int_{t}^{\mathrm{T}} \mathrm{d} t_{1} \widehat{\mathcal{G}}_{1}\left(t, t_{1}\right) \widehat{\mathcal{P}}_{0}\left(t, t_{1}\right) \int_{t_{1}}^{\mathrm{T}} \mathrm{d} t_{2} \widehat{\mathcal{G}}_{1}\left(t_{1}, t_{2}\right) \widehat{\mathcal{P}}_{0}\left(t_{1}, t_{2}\right) \widehat{\mathcal{P}}_{0}\left(t_{2}, \mathrm{~T}\right) \varphi \\
& +\int_{t}^{\mathrm{T}} \mathrm{d} t_{1} \widehat{\mathcal{G}}_{2}\left(t, t_{1}\right) \widehat{\mathcal{P}}_{0}\left(t, t_{1}\right) \widehat{\mathcal{P}}_{0}\left(t_{1}, \mathrm{~T}\right) \varphi \\
& =\left(\int_{t}^{\mathrm{T}} \mathrm{d} t_{1} \widehat{\mathcal{G}}_{2}\left(t, t_{1}\right)+\int_{t}^{\mathrm{T}} \mathrm{d} t_{1} \int_{t_{1}}^{\mathrm{T}} \mathrm{d} t_{2} \widehat{\mathcal{G}}_{1}\left(t, t_{1}\right) \widehat{\mathcal{G}}_{1}\left(t_{1}, t_{2}\right)\right) p_{0}(t) .
\end{aligned}
$$

\section{Approximation formulas in Section 5}

Recall that it is assumed in the following computations that if an operator is followed by nothing, it acts on the constant 1 .

- Approximation formula for $\Lambda$ in Remark 1:

In the first order correction (31), we get

$$
\begin{aligned}
& \int_{t}^{\mathrm{T}} \mathrm{d} t_{1}\left(\frac{1}{2} \lambda^{2}\right)_{1}\left(\widetilde{X}\left(t, t_{1}\right), \widetilde{y}\left(t, t_{1}\right)\right) \\
& =\int_{t}^{\mathrm{T}} \mathrm{d} t_{1}\left[\left(\frac{1}{2} \lambda^{2}\right)_{1,0}\left(x-\bar{x}-\left(\frac{1}{2} \sigma^{2}\right)_{0}\left(t_{1}-t\right)\right)+\left(\frac{1}{2} \lambda^{2}\right)_{0,1}\left(y-\bar{y}+(c-\rho \beta \lambda)_{0}\left(t_{1}-t\right)\right)\right] \\
& =\left(\frac{1}{2} \lambda^{2}\right)_{1,0}\left((x-\bar{x})(\mathrm{T}-t)-\left(\frac{1}{2} \sigma^{2}\right)_{0} \frac{1}{2}(\mathrm{~T}-t)^{2}\right)+\left(\frac{1}{2} \lambda^{2}\right)_{0,1}\left((y-\bar{y})(\mathrm{T}-t)+(c-\rho \beta \lambda)_{0} \frac{1}{2}(\mathrm{~T}-t)^{2}\right) .
\end{aligned}
$$

Similarly, in the second order correction (32), we compute the following term

$$
\begin{aligned}
& \int_{t}^{\mathrm{T}} \mathrm{d} t_{1} \widetilde{\mathfrak{G}}_{1}\left(t, t_{1}\right) \int_{t_{1}}^{\mathrm{T}} \mathrm{d} t_{2}\left(\frac{1}{2} \lambda^{2}\right)_{1}\left(\widetilde{x}\left(t, t_{2}\right), \tilde{y}\left(t, t_{2}\right)\right) \\
& =-\left(\frac{1}{2} \lambda^{2}\right)_{1,0} \frac{1}{2}(\mathrm{~T}-t)^{2}\left(\left(\left(\frac{1}{2} \sigma^{2}\right)_{1,0}(x-\bar{x})+\left(\frac{1}{2} \sigma^{2}\right)_{0,1}(y-\bar{y})\right)\right. \\
& \left.\quad+\frac{1}{3}(\mathrm{~T}-t)\left(-\left(\frac{1}{2} \sigma^{2}\right)_{1,0}\left(\frac{1}{2} \sigma^{2}\right)_{0}+\left(\frac{1}{2} \sigma^{2}\right)_{0,1}(c-\rho \beta \lambda)_{0}\right)\right) \\
& +\left(\frac{1}{2} \lambda^{2}\right)_{0,1} \frac{1}{2}(\mathrm{~T}-t)^{2}\left(\left((c-\rho \beta \lambda)_{1,0}(x-\bar{x})+(c-\rho \beta \lambda)_{0,1}(y-\bar{y})\right)\right. \\
& \left.\quad+\frac{1}{3}(\mathrm{~T}-t)\left(-(c-\rho \beta \lambda)_{1,0}\left(\frac{1}{2} \sigma^{2}\right)_{0}+(c-\rho \beta \lambda)_{0,1}(c-\rho \beta \lambda)_{0}\right)\right) .
\end{aligned}
$$

In the next term, we have

$$
\begin{aligned}
& \int_{t}^{\mathrm{T}} \mathrm{d} t_{1}\left(\frac{1}{2} \lambda^{2}\right)_{2}\left(\widetilde{x}\left(t, t_{1}\right), \widetilde{y}\left(t, t_{1}\right)\right) \\
& =\frac{1}{2}\left(\frac{1}{2} \lambda^{2}\right)_{2,0}\left((x-\bar{x})^{2}(\mathrm{~T}-t)-(\mathrm{T}-t)^{2}(x-\bar{x})\left(\frac{1}{2} \sigma^{2}\right)_{0}+\frac{1}{3}(\mathrm{~T}-t)^{3}\left(\left(\frac{1}{2} \sigma^{2}\right)_{0}\right)^{2}\right) \\
& +\left(\frac{1}{2} \lambda^{2}\right)_{1,1}\left((\mathrm{~T}-t)(x-\bar{x})(y-\bar{y})+\frac{(\mathrm{T}-t)^{2}}{2}\left((x-\bar{x})(c-\rho \beta \lambda)_{0}-(y-\bar{y})\left(\frac{1}{2} \sigma^{2}\right)_{0}\right)-\frac{(\mathrm{T}-t)^{3}}{3}(c-\rho \beta \lambda)_{0}\left(\frac{1}{2} \sigma^{2}\right)_{0}\right) \\
& +\frac{1}{2}\left(\frac{1}{2} \lambda^{2}\right)_{0,2}\left((y-\bar{y})^{2}(\mathrm{~T}-t)+(\mathrm{T}-t)^{2}(y-\bar{y})(c-\rho \beta \lambda)_{0}+\left((c-\rho \beta \lambda)_{0}\right)^{2} \frac{1}{3}(\mathrm{~T}-t)^{3}\right) .
\end{aligned}
$$


In the final term, we have

$$
\begin{aligned}
& \int_{t}^{\mathrm{T}} \mathrm{d} t_{1}\left(\frac{1}{2} \lambda^{2}\right)_{0,1}\left(\mathrm{~T}-t_{1}\right) \partial_{y}\left(\int_{t_{1}}^{\mathrm{T}} \mathrm{d} t_{2} \widetilde{\mathcal{G}}_{1}\left(t, t_{2}\right)\right) p^{\mathrm{BS}}(t) \\
& =\int_{t}^{\mathrm{T}} \mathrm{d} t_{1}\left(\frac{1}{2} \lambda^{2}\right)_{0,1}\left(\mathrm{~T}-t_{1}\right)^{2}\left(\frac{1}{2} \sigma^{2}\right)_{0,1}\left(\partial_{x}^{2}-\partial_{x}\right) p^{\mathrm{BS}}(t) \\
& =\int_{t}^{\mathrm{T}} \mathrm{d} t_{1}\left(\frac{1}{2} \lambda^{2}\right)_{0,1}\left(\mathrm{~T}-t_{1}\right)^{2}\left(\frac{1}{2} \sigma^{2}\right)_{0,1} \frac{1}{\sigma_{0} \sqrt{\mathrm{T}-t}} \mathrm{e}^{x} \phi\left(d_{+}\left(t, x ; \sigma_{0}\right)\right) \\
& =\left(\frac{1}{2} \lambda^{2}\right)_{0,1}\left(\frac{1}{2} \sigma^{2}\right)_{0,1} \frac{1}{\sigma_{0} \sqrt{\mathrm{T}-t}} \mathrm{e}^{x} \phi\left(d_{+}\left(t, x ; \sigma_{0}\right)\right) \frac{(\mathrm{T}-t)^{3}}{3}
\end{aligned}
$$

- Approximation formula for option price $p$ under the minimal martingale measure of Remark 1:

The first order correction term (28) is given as

$$
\begin{aligned}
p_{1}(t)= & \int_{t}^{\mathrm{T}} \mathrm{d} t_{1} \widetilde{\mathcal{G}}_{1}\left(t, t_{1}\right) p^{\mathrm{BS}}(t) \\
= & \left(\frac{1}{2} \sigma^{2}\right)_{1,0}\left((x-\bar{x})(\mathrm{T}-t)+\left(-\left(\frac{1}{2} \sigma^{2}\right)_{0}\right) \frac{(\mathrm{T}-t)^{2}}{2}\right)\left(\partial_{x}^{2}-\partial_{x}\right) p^{\mathrm{BS}}(t) \\
& +\left(\frac{1}{2} \sigma^{2}\right)_{0,1}\left((y-\bar{y})(\mathrm{T}-t)+(c-\rho \beta \lambda)_{0} \frac{(\mathrm{T}-t)^{2}}{2}\right)\left(\partial_{x}^{2}-\partial_{x}\right) p^{\mathrm{BS}}(t) \\
& +\frac{(\mathrm{T}-t)^{2}}{2}\left(2\left(\frac{1}{2} \sigma^{2}\right)_{1,0}\left(\frac{1}{2} \sigma^{2}\right)_{0}+\left(\frac{1}{2} \sigma^{2}\right)_{0,1}(\rho \sigma \beta)_{0}\right) \partial_{x}\left(\partial_{x}^{2}-\partial_{x}\right) p^{\mathrm{BS}}(t) .
\end{aligned}
$$

The second order correction term (29) writes as

$$
p_{2}(t)=\left(\int_{t}^{\mathrm{T}} \mathrm{d} t_{1} \widetilde{\mathcal{G}}_{2}\left(t, t_{1}\right)+\int_{t}^{\mathrm{T}} \mathrm{d} t_{1} \int_{t_{1}}^{\mathrm{T}} \mathrm{d} t_{2} \widetilde{\mathcal{G}}_{1}\left(t, t_{1}\right) \widetilde{\mathcal{G}}_{1}\left(t_{1}, t_{2}\right)\right) p^{\mathrm{BS}}(t) .
$$

In the first expression above, we get

$$
\begin{aligned}
& \int_{t}^{\mathrm{T}} \mathrm{d} t_{1} \widetilde{\mathcal{G}}_{2}\left(t, t_{1}\right) p^{\mathrm{BS}}(t) \\
= & \int_{t}^{\mathrm{T}} \mathrm{d} t_{1}\left[\frac{1}{2}\left(\frac{1}{2} \sigma^{2}\right)_{2,0}\left(X\left(t, t_{1}\right)-\bar{x}\right)^{2}+\frac{1}{2}\left(\frac{1}{2} \sigma^{2}\right)_{0,2}\left(y\left(t, t_{1}\right)-\bar{y}\right)^{2}+\left(\frac{1}{2} \sigma^{2}\right)_{1,1}\left(X\left(t, t_{1}\right)-\bar{x}\right)\left(y\left(t, t_{1}\right)-\bar{y}\right)\right] \\
& \times\left(\partial_{x}^{2}-\partial_{x}\right) p^{\mathrm{BS}}(t) .
\end{aligned}
$$

Since in both Heston and reciprocal Heston model considered in Section 5.1 and 5.2, respectively, $\frac{1}{2} \sigma^{2}=\frac{y}{2}$, we get $\left(\frac{1}{2} \sigma^{2}\right)_{2,0}=\left(\frac{1}{2} \sigma^{2}\right)_{0,2}=\left(\frac{1}{2} \sigma^{2}\right)_{1,1}=0$. Thus, the second order correction term in the Heston and reciprocal Heston model is given as

$$
\begin{aligned}
& p_{2}(t) \\
= & \int_{t}^{\mathrm{T}} \mathrm{d} t_{1} \int_{t_{1}}^{\mathrm{T}} \mathrm{d} t_{2} \widetilde{\mathfrak{G}}_{1}\left(t, t_{1}\right) \widetilde{\mathcal{G}}_{1}\left(t_{1}, t_{2}\right) p^{\mathrm{BS}}(t) \\
= & \int_{t}^{\mathrm{T}} \mathrm{d} t_{1} \int_{t_{1}}^{\mathrm{T}} \mathrm{d} t_{2}\left(\frac{1}{2} \sigma^{2}\right)_{0,1}\left(y\left(t, t_{1}\right)-\bar{y}\right)\left(\frac{1}{2} \sigma^{2}\right)_{0,1}\left(y\left(t_{1}, t_{2}\right)-\bar{y}\right)\left(\partial_{x}^{2}-\partial_{x}\right)\left(\partial_{x}^{2}-\partial_{x}\right) p^{\mathrm{BS}}(t) \\
= & \frac{1}{4} \int_{t}^{\mathrm{T}} \mathrm{d} t_{1} \int_{t_{1}}^{\mathrm{T}} \mathrm{d} t_{2}\left(y-\bar{y}+\left(t_{1}-t\right)\left((c-\rho \beta \lambda)_{0}+(\rho \sigma \beta)_{0} \partial_{x}\right)\right)\left(y-\bar{y}+\left(t_{2}-t_{1}\right)\left((c-\rho \beta \lambda)_{0}+(\rho \sigma \beta)_{0} \partial_{x}\right)\right) \\
& \times\left(\partial_{x}^{2}-\partial_{x}\right)\left(\partial_{x}^{2}-\partial_{x}\right) p^{\mathrm{BS}}(t) .
\end{aligned}
$$

We get the following three terms from above: 

a. $\frac{(\mathrm{T}-t)^{2}}{8}(y-\bar{y})^{2}\left(\partial_{x}^{2}-\partial_{x}\right)^{2} p^{\mathrm{BS}}(t)$
b. $\frac{(\mathrm{T}-t)^{3}}{12}(y-\bar{y})\left((c-\rho \beta \lambda)_{0}+(\rho \sigma \beta)_{0} \partial_{x}\right)\left(\partial_{x}^{2}-\partial_{x}\right)^{2} p^{\mathrm{BS}}(t)$
c. $\frac{(\mathrm{T}-t)^{4}}{96}\left((c-\rho \beta \lambda)_{0}+(\rho \sigma \beta)_{0} \partial_{x}\right)^{2}\left(\partial_{x}^{2}-\partial_{x}\right)^{2} p^{\mathrm{BS}}(t)$

In the formulas above, we need to compute

$$
\partial_{x}^{n}\left(\partial_{x}^{2}-\partial_{x}\right) p^{\mathrm{BS}}(t), n \in\{0\} \cup \mathbb{N},
$$

which can be obtained from Equation (C.4) in Appendix C of Lorig (2018). We can use the result

$$
\left(\partial_{x}^{2}-\partial_{x}\right) p^{\mathrm{BS}}\left(\sigma_{0}\right)=\frac{1}{\sigma_{0} \sqrt{2 \pi \tau}} \mathrm{e}^{-z^{2}+k}, \quad z:=\frac{x-k-\frac{1}{2} \sigma_{0}^{2} \tau}{\sigma_{0} \sqrt{2 \tau}}, \quad \tau:=\mathrm{T}-t,
$$

with

$$
\frac{\partial_{x}^{n}\left(\partial_{x}^{2}-\partial_{x}\right) p^{\mathrm{BS}}\left(\sigma_{0}\right)}{\left(\partial_{x}^{2}-\partial_{x}\right) p^{\mathrm{BS}}\left(\sigma_{0}\right)}=\mathrm{e}^{z^{2}} \partial_{x}^{n} \mathrm{e}^{-z^{2}}=\left(\frac{-1}{\sigma_{0} \sqrt{2 \pi \tau}}\right)^{n} h_{n}(z)
$$

where $h_{n}(z):=(-1)^{n} \mathrm{e}^{z^{2}} \partial_{x}^{n} \mathrm{e}^{-z^{2}}$ is the $n$-th Hermite polynomial, to compute the terms in (40). 